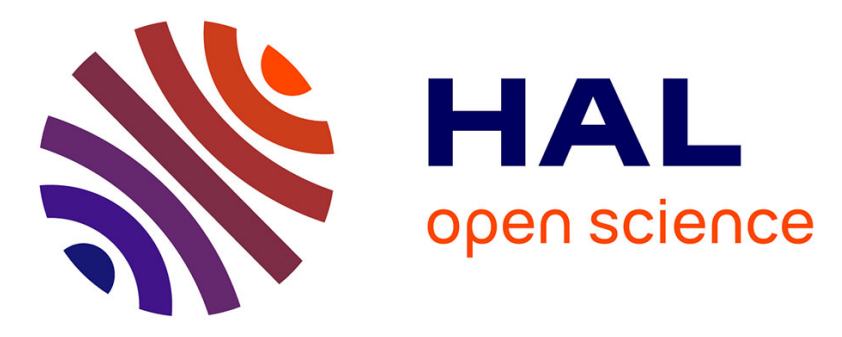

\title{
Dissipation inequality-based periodic homogenization of wavy materials
}

Dimitrios Tsalis, Georges Chatzigeorgiou, Charalampos Tsakmakis, Nicolas

Charalambakis

\section{- To cite this version:}

Dimitrios Tsalis, Georges Chatzigeorgiou, Charalampos Tsakmakis, Nicolas Charalambakis. Dissipation inequality-based periodic homogenization of wavy materials. Composites Part B: Engineering, 2015, 76, pp.89-104. 10.1016/j.compositesb.2015.02.014 . hal-01201913

\section{HAL Id: hal-01201913 \\ https://hal.science/hal-01201913}

Submitted on 18 Sep 2015

HAL is a multi-disciplinary open access archive for the deposit and dissemination of scientific research documents, whether they are published or not. The documents may come from teaching and research institutions in France or abroad, or from public or private research centers.
L'archive ouverte pluridisciplinaire HAL, est destinée au dépôt et à la diffusion de documents scientifiques de niveau recherche, publiés ou non, émanant des établissements d'enseignement et de recherche français ou étrangers, des laboratoires publics ou privés. 


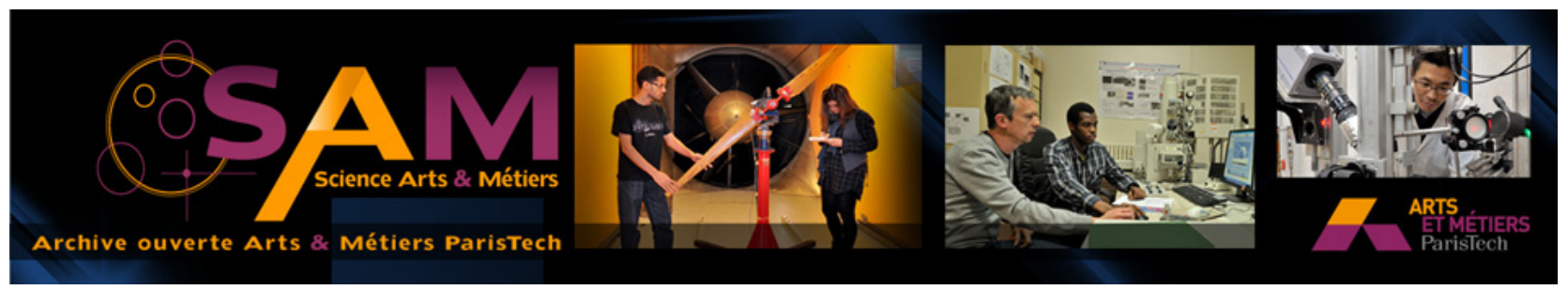

\section{Science Arts \& Métiers (SAM)}

is an open access repository that collects the work of Arts et Métiers ParisTech researchers and makes it freely available over the web where possible.

This is an author-deposited version published in: http://sam.ensam.eu

Handle ID: .http://hdl.handle.net/10985/10050

\section{To cite this version :}

Dimitrios TSALIS, George CHATZIGEORGIOU, Charalampos TSAKMAKIS, Nicolas CHARALAMBAKIS - Dissipation inequality-based periodic homogenization of wavy materials Composites Part B - Vol. 76, p.89-104 - 2015 


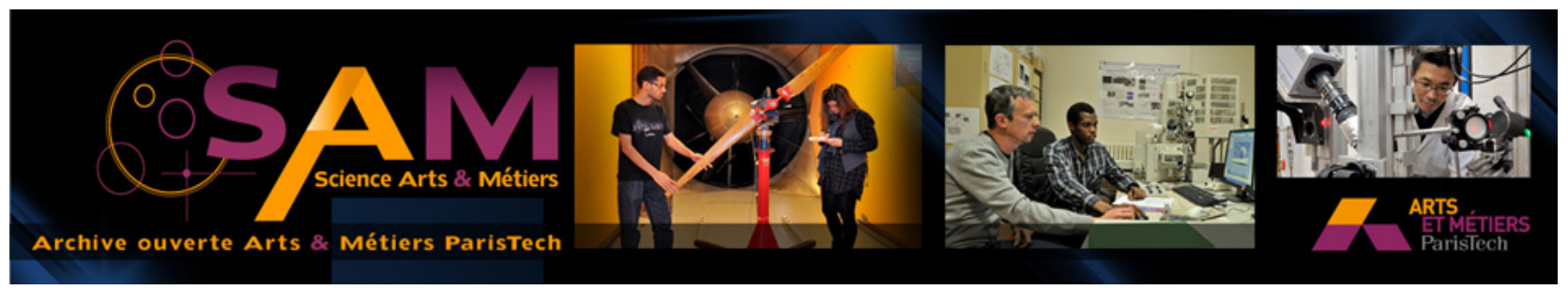

Science Arts \& Métiers (SAM)

is an open access repository that collects the work of Arts et Métiers ParisTech researchers and makes it freely available over the web where possible.

This is an author-deposited version published in: http://sam.ensam.eu Handle ID: .http://hdl.handle.net/null

\section{To cite this version :}

Dimitrios TSALIS, George CHATZIGEORGIOU, Charalampos TSAKMAKIS, Nicolas CHARALAMBAKIS - Dissipation inequality-based periodic homogenization of wavy materials Composites Part B - Vol. 76, p.89-104 - 2015 


\title{
Dissipation inequality-based periodic homogenization of wavy materials
}

\author{
Dimitrios Tsalis a , George Chatzigeorgiou ${ }^{\text {b, }}{ }^{*}$, Charalampos Tsakmakis ${ }^{\text {c }}$ \\ Nicolas Charalambakis ${ }^{\text {a }}$ \\ a Department of Civil Engineering, Aristotle University of Thessaloniki, GR 54124 Thessaloniki, Greece \\ b LEM3-UMR 7239 CNRS, Arts et Metiers ParisTech Metz-Lorraine, 4 Rue Augustin Fresnel, 57078 Metz, France \\ ${ }^{\mathrm{c}}$ Technische Universitat Darmstadt, Institut fur Mechanik, Hochschulstrasse 1, 64289 Darmstadt, Germany
}

\begin{abstract}
A B S T R A C T
In this paper we present an internal variable-based homogenization of a composite made of wavy elasticperfectly plastic layers. In the context of a strain-driven process, the macrostress and the effective yield surface are expressed in terms of the residual stresses, which act as hardening parameters in the effective behavior of the composite. Moreover, an approximate two-steps homogenization scheme useful for composites made of matrix with wavy inclusions is proposed and a comparison with one computational and one semi-analytical homogenization method is presented.
\end{abstract}

\section{Introduction}

Modeling the mechanical behavior of non-linear heterogeneous materials has been the subject of many research papers from both mathematical and computational point of view $[4,6,9,9-11,13,16,22,25,29,35,36,38-42,49-51]$. Special attention has been paid to the case of composites with properties and/or geometry dependent on a non-linear periodicity function $[5,7,8,14,15]$, and/or non-linear constitutive behavior of the constituent materials $[14,15,52,53]$. For general plasticity equations and viscoplasticity with non-linear hardening we refer to the book [1]; which covers constitutive equations of "monotone type", and to [2]

The role of dissipation inequality in homogenization of dissipative materials is crucial: it needs to be considered in both microand macro-level in order to lead to the correct constitutive evolution equations relating stress and internal variables. The local problem in generalized standard materials (GSM) was completely described by Refs. $[30-34,43,47]$ (see also [17,18,20], based on the fundamental works of [21] and [37]. Generalized materials are described by state and internal variables. Generalized forces are then defined from the free energy function expression in terms of the above variables. Additionally, the dissipation inequality holds and by the Lagrange multiplier's technique gives the evolutionary equations.

Wavy architectures can be found in the nature or constructed for functional purposes or accidently obtained in manufacturing processes and the thermomechanical behavior of forming materials or structures under specific loading and environmental conditions is of special technological interest [23-26,54]. Wavy multilayer materials and structural components are characterized by a wavy periodicity at several scales: corrugated cross sections used to stiffen structural panels, laminated composite plates exhibiting manufacturing induced waviness with problematic behavior under compression, microstructures with wavy architectures, biological tissues such as chordea tendenea found in heart valves, where stiff collagen fibril crimp patterns control the opening and closing of the valve leaflets [24], continue to form the subject of intense research effort [22,25-28,45,54-56]. In nanotechnology, wavy interfacial morphology can enhance the overall properties of composites made of thin metallic and ceramic multilayers for magnetic, optoelectronic and high-speed electronic applications [24]. Novel fuzzy fiber reinforced composites are composed of carbon fibers, wavy carbon nanotubes and epoxy matrix, with the carbon fibers radially grown on the circumferential surfaces of the carbon fibers $[14,15,28]$. 
The objective of this paper is twofold: first, to present an analytical dissipation inequality-based homogenization scheme for a wavy multilayered medium made of elastic-perfectly plastic components; second, to propose an approximate two-steps homogenization for a composite made of a matrix with wavy inclusions. In Section 2 a review on the role of dissipation inequality in the homogenization process of rate-independent dissipative materials is presented. More specifically, it is verified that the overall behavior of a heterogeneous material is a generalized standard material behavior and the strain-driven localization problem is formulated. Moreover, the fundamental assumptions, of additivity for the free energy and of dependence of its effective value on the microstrain and a finite number of micro-internal parameters, allow for defining the effective generalized forces through the variation of the effective energy, and subsequently of expressing the overall dissipation starting from the microstresses and the rates of internal micro-hardening "forces" and microplastic strains, in correlation with micro-yield surface. In Section 3, the analytical homogenization of a wavy layered composite made of two elastic-perfectly plastic materials is presented. This includes the analytical expressions for the effective constitutive law, for the macroscopic yield surface and for the residual microstresses in terms of the macrostrain and the plastic microstrains. An interesting finding is that, as expected [49], even if the constituents are isotropic and without hardening, the composite exhibits anisotropy and hardening due to the presence of residual stresses in the effective yield surface. Finally, in Section 4, a two-steps approximate homogenization scheme is presented for a composite with wavy inclusions and numerical examples of the proposed homogenization scheme are presented, corresponding to a unit cell under monotone and cyclic loading respectively. Moreover, the results are compared to one semi-analytical and one computational (Finite Volume Direct Averaging Micromechanics-FVDAM [41], method. The construction of the effective yield surface completes the numerical experiments. In three appendices, all matrices needed for the analytical expressions of micro-and macrovariables are presented.

\section{The dissipation in heterogeneous generalized standard materials}

We consider three spatial variables that coexist for the description of the problem. The first one is the macroscale denoted by $^{1} \boldsymbol{x}$ in the heterogeneous body, at which the heterogeneities, characterized by $\varepsilon$, are very small compared to the whole structure and can be considered as invisible. The second spatial variable is the microscale denoted by $\frac{\boldsymbol{x}}{\boldsymbol{s}}$, which is the scale for the heterogeneities (Fig. 1). The third spatial variable is used only if the body exhibits a generalized (non-linear) periodicity.

The case of materials with generalized periodicity is of special interest for two reasons: first, since it corresponds sometimes to a non-repetitive geometry as in composites with cylindrical periodicity and second, it uses simpler unit cells and may allow semianalytical homogenization methods [53]. The choice of the representative volume element is made with respect to the generalized periodicity vector function $\mathbf{Q}(\boldsymbol{x})$ and $Y=\left[0, y_{1}\right] \times\left[0, y_{2}\right] \times\left[0, y_{3}\right]$ is chosen to be the basic cell, where

$\boldsymbol{y}=\frac{\mathrm{Q}(\boldsymbol{x})}{\varepsilon}$

\footnotetext{
${ }^{1}$ In the sequel, every vector or tensor will be denoted with two ways: a boldsymbol or its indicial notation. The scalar quantities appear in regular fonts.
}

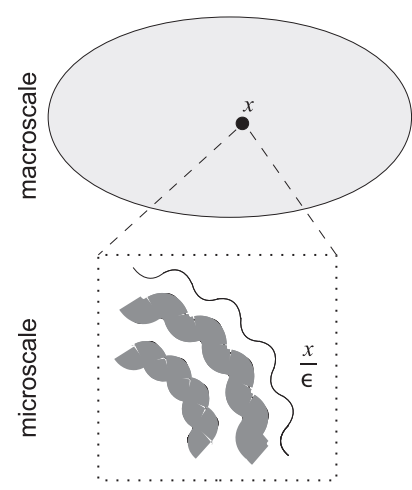

Fig. 1. Macro- and microscale.

The dependence of functions on the microcoordinate is performed (in a non-periodic way, except if $\boldsymbol{\varrho}(\boldsymbol{x})=\boldsymbol{x}$ ) via

$\overline{\boldsymbol{y}}=\frac{\boldsymbol{x}}{\varepsilon}$.

In this paper we focus our attention on the multilayered materials (see Fig. 2). For simplicity, we present the case of structures with layers parallel to the $x_{3}$-axis. Thus, at every macropoint $\left(x_{1}, x_{2}\right)$ microstress and microstrain are uniform in every phase with values depending on $\left(x_{1}, x_{2}\right)$ [52]. More specifically, the angle $\theta\left(x_{1}, x_{2}\right)$ of the tangent at the macropoint with $x_{1}$-axis enters the equations of microstress equilibrium and the equations of continuity at the interfaces, as well as the effective tangent modulus at $\left(x_{1}, x_{2}\right)$.

Let us now denote field variables $\sigma^{0}, \boldsymbol{\varepsilon}^{0}$ and $\boldsymbol{u}^{1}$ as microscopic variables and $\boldsymbol{\Sigma}, \boldsymbol{E}$ and $\boldsymbol{u}^{0}$ as the macroscopic variables. The macroscopic quantities depend only on the macrocoordinate $\boldsymbol{x}$. It is worth noticing that both classes of deformation fields are related to the representative volume element located at $\boldsymbol{x}$. Away from the boundaries, stress and strain fields conform at the microlevel to the generalized periodicity conditions:

$\boldsymbol{\sigma}^{0}, \boldsymbol{\varepsilon}^{0}$ are $Y$ - periodic functions of $\boldsymbol{y}$.

The actual displacement $\boldsymbol{u}^{0}$ within $Y$ located at $\boldsymbol{x}$ is assumed to be expressed as a sum of a linear and a generalized-periodic part $[48,50,52]$.

$u_{i}^{0}(\boldsymbol{x}, \overline{\boldsymbol{y}}, \boldsymbol{y})=E_{i j} \bar{y}_{j}+u_{i}^{1}$,

where

$u_{i}^{1}=u_{i}^{1}(\boldsymbol{x}, \boldsymbol{y})$

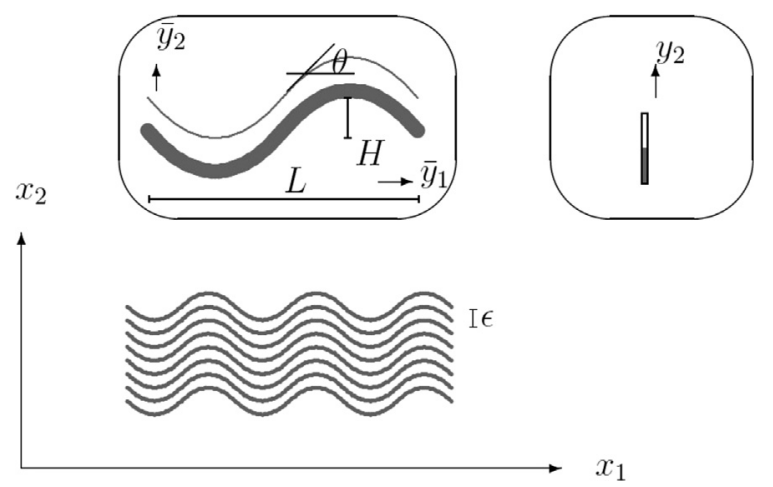

Fig. 2. Wavy multilayered material. 
is periodic with respect to $\boldsymbol{y}$. Microstrain is then defined from (2.4) with respect to the microcoordinate,

$\left.\left.\varepsilon_{i j}^{0}=\operatorname{sym} \frac{\partial u_{i}^{0}}{\partial \bar{y}_{j}}\right)=\frac{1}{2} \frac{\partial u_{i}^{0}}{\partial \bar{y}_{j}}+\frac{\partial u_{j}^{0}}{\partial \bar{y}_{i}}\right)$.

while the macrostrain is defined with respect to the macrocoordinate,

$\left.\left.E_{i j}=\operatorname{sym} \frac{\partial u_{i}^{0}}{\partial x_{j}}\right)=\frac{1}{2} \frac{\partial u_{i}^{0}}{\partial x_{j}}+\frac{\partial u_{j}^{0}}{\partial x_{i}}\right)$.

In Ref. [52] it was shown that

$\left.\varepsilon_{i j}^{0}=E_{i j}+\frac{1}{2} \frac{\partial u_{i}^{1}}{\partial y_{k}} \frac{\partial \varrho_{k}}{\partial x_{j}}+\frac{\partial u_{j}^{1}}{\partial y_{k}} \frac{\partial \varrho_{k}}{\partial x_{i}}\right)$

and

$\left\langle\varepsilon_{i j}^{0}\right\rangle=\frac{1}{|Y|} \int_{Y} \varepsilon_{i j}^{0} d Y=E_{i j}$.

As it was proved in Ref. [52] the microstress satisfies the equation of equilibrium

$\frac{\partial \sigma_{i j}^{0}}{\partial \bar{y}_{j}}=\frac{\partial \sigma_{i j}^{0}}{\partial y_{m}} \frac{\partial \varrho_{m}}{\partial x_{j}}=0$

under periodicity conditions.

Concerning the macrostress, this is defined as the mean value of the microstress over the unit cell,

$\Sigma_{i j}=\left\langle\sigma_{i j}^{0}\right\rangle=\frac{1}{|Y|} \int_{Y} \sigma_{i j}^{0} d Y$.

In Ref. [52] it was shown that microstress and microstrain (i) satisfy the Mandel-Hill's lemma

$\left\langle\sigma_{i j}^{0} \varepsilon_{i j}^{0}\right\rangle=\Sigma_{i j} E_{i j}$.

and (ii) are the two-scale convergence limits [3] of the heterogeneous problem over the whole structure.

For the moment we assume that the material is a homogeneous generalized standard material (GSM, [46]. In classical rate-independent plasticity the free energy $\psi$ is a function of the internal variables

$\psi=\psi(\boldsymbol{\varepsilon}, \boldsymbol{a})$,

where $(\boldsymbol{\varepsilon}, \boldsymbol{a})$ are the state variables, $\boldsymbol{\varepsilon}=\boldsymbol{\varepsilon}^{e}+\boldsymbol{\varepsilon}^{p}$ is the strain tensor decomposed into an elastic and a plastic part, while $\boldsymbol{a}=\left(\boldsymbol{\varepsilon}^{p}, \boldsymbol{\xi}\right)$ are the internal variables. Here we assume that the internal variables are the plastic strain tensor $\varepsilon^{p}$ and a hardening vector $\xi$. We also assume that the free energy is decomposed into an elastic part (stored energy) $W=1 / 2\left(\boldsymbol{\varepsilon}-\boldsymbol{\varepsilon}^{p}\right): \boldsymbol{C}:\left(\boldsymbol{\varepsilon}-\boldsymbol{\varepsilon}^{p}\right)$ and a plastic part $H^{p}(\boldsymbol{\xi})$ which for simplicity is assumed to be independent of the plastic strain,

$\psi(\boldsymbol{\varepsilon}, \boldsymbol{a})=\frac{1}{2}\left(\boldsymbol{\varepsilon}-\boldsymbol{\varepsilon}^{p}\right): \boldsymbol{C}:\left(\boldsymbol{\varepsilon}-\boldsymbol{\varepsilon}^{p}\right)+H^{p}(\boldsymbol{\xi})$.

Then, the generalized forces $\boldsymbol{A}=\left(\boldsymbol{A}^{p}, \boldsymbol{A}^{\xi}\right)$, associated to the internal variables $\boldsymbol{a}$, are given by
$\boldsymbol{A}^{p}=-\frac{\partial \psi}{\partial \boldsymbol{\varepsilon}^{p}}=\boldsymbol{\sigma}, \quad \boldsymbol{A}^{\xi}=-\frac{\partial \psi}{\partial \xi}=\boldsymbol{q}$,

where $\boldsymbol{\sigma}$ is the stress tensor and $\boldsymbol{q}$ is the internal hardening "force". Thus the plastic criterion can be written

$f\left(\boldsymbol{A}^{p}, \boldsymbol{A}^{\xi}\right) \leq 0$.

Additionally, the dissipation function is given by

$$
\begin{aligned}
d & \left.=\boldsymbol{\sigma}: \dot{\boldsymbol{\varepsilon}}-\dot{\psi}=\boldsymbol{\sigma}: \dot{\boldsymbol{\varepsilon}}-\frac{\partial \psi}{\partial \boldsymbol{\varepsilon}}:\left(\dot{\boldsymbol{\varepsilon}}-\boldsymbol{\varepsilon}^{p}\right)+\frac{\partial \psi}{\partial \xi} \cdot \dot{\xi}\right) \\
& =\boldsymbol{\sigma}: \dot{\boldsymbol{\varepsilon}}^{p}-\frac{\partial \psi}{\partial \boldsymbol{\xi}} \cdot \dot{\boldsymbol{\xi}}=\boldsymbol{A}^{p}: \dot{\boldsymbol{\varepsilon}}^{p}+\boldsymbol{A}^{\xi} \cdot \dot{\boldsymbol{\xi}}=\boldsymbol{A}: \dot{\boldsymbol{a}} .
\end{aligned}
$$

The dissipation inequality is written

$d \geq 0$ for every $\boldsymbol{A}^{p}, \boldsymbol{A}^{\xi}$ satisfying $f\left(\boldsymbol{A}^{p}, \boldsymbol{A}^{\xi}\right) \leq 0$.

Then the normality law is obtained simply from Eq. (2.18) by minimizing

$-d+\dot{\lambda} f\left(\boldsymbol{A}^{p}, \boldsymbol{A}^{\xi}\right)$

over $\boldsymbol{A}^{p}, \boldsymbol{A}^{\xi}$, where $\lambda$ is a Lagrange multiplier. Therefore the problem of determination of the evolution equations leads to the problem of minimization of the Lagrangian

$\min 2\left(\boldsymbol{A}^{p}, \boldsymbol{A}^{\xi}\right)=-\boldsymbol{A}^{p}: \dot{\boldsymbol{\varepsilon}}^{p}-\boldsymbol{A}^{\xi} \cdot \dot{\xi}+\dot{\lambda} f\left(\boldsymbol{A}^{p}, \boldsymbol{A}^{\xi}\right)$

from which taking the first variation with respect to $\boldsymbol{A}^{p}, \boldsymbol{A}^{\xi}$ respectively yields the evolutionary equations

$\dot{\boldsymbol{\varepsilon}}^{p}=\dot{\lambda} \frac{\partial f}{\partial \boldsymbol{A}^{p}}, \quad \dot{\boldsymbol{\xi}}=\dot{\lambda} \frac{\partial f}{\partial \boldsymbol{A}^{\xi}}$.

These equations must be supplemented by the Kuhn-Tucker conditions

$f \leq 0, \quad \dot{\lambda} \geq 0, \quad f \dot{\lambda}=0$

Let us now pass to the verification that the overall behavior of a heterogeneous material is a GSM behavior $[37,49]$. We consider the strain-driven localization problem: for given macrostrain $\boldsymbol{E}$ and internal parameters $\boldsymbol{a}^{0}$ in the unit cell $Y$, find the relation between macrostress $\boldsymbol{\Sigma}$ and macrostrain $\boldsymbol{E}$ if

$\boldsymbol{\varepsilon}^{0}=\operatorname{sym} \nabla \boldsymbol{u}^{0}$ in $Y,\left\langle\boldsymbol{\varepsilon}^{0}\right\rangle=\boldsymbol{E}, \operatorname{div}_{\overline{\boldsymbol{y}}} \boldsymbol{\sigma}^{0}=0$ in $Y,\left\langle\boldsymbol{\sigma}^{0}\right\rangle=\boldsymbol{\Sigma}$,

$\boldsymbol{\sigma}^{0}=\frac{\partial \psi^{0}\left(\boldsymbol{\varepsilon}^{0}, \boldsymbol{a}^{0}\right)}{\partial \boldsymbol{\varepsilon}^{0}}, \psi^{0}\left(\boldsymbol{\varepsilon}^{0}, \boldsymbol{a}^{0}\right)=\frac{1}{2}\left(\boldsymbol{\varepsilon}^{0}-\boldsymbol{\varepsilon}^{p 0}\right): \boldsymbol{C}^{0}:\left(\boldsymbol{\varepsilon}^{0}-\boldsymbol{\varepsilon}^{p 0}\right)+H^{p 0}\left(\xi^{0}\right)$,

$\boldsymbol{\sigma}^{0}$ and $\boldsymbol{\varepsilon}^{0}$ periodic on $\partial Y$.

We note that, for this verification, special form Eq. (2.4) of the displacement $u^{0}$ is not needed. In Ref. [49] it was shown that this is an elastic problem giving the solution

$\boldsymbol{\sigma}^{0}=\boldsymbol{\sigma}^{0}\left(\boldsymbol{E}, \boldsymbol{a}^{0}\right), \boldsymbol{\varepsilon}^{0}=\boldsymbol{\varepsilon}^{0}\left(\boldsymbol{E}, \boldsymbol{a}^{0}\right), \quad \boldsymbol{\Sigma}=\left\langle\boldsymbol{\sigma}^{0}\left(\boldsymbol{E}, \boldsymbol{a}^{0}\right)\right\rangle$.

Consequently, microstress and microstrain can be obtained from a strain-driven cell problem as functions of the macrostrain and the internal variables. The main difficulty is that $a^{0}$ must be reduced to a finite-dimensional field, and this issue is the main subject of the 
computational methods developed by Fish, Dvorak, Suquet and other researchers [17-19,30,31,34]. In order to determine the effective constitutive behavior of the composite, we assume that the effective free energy satisfies both relations

$\Psi=\left\langle\psi^{0}\left(\boldsymbol{\varepsilon}^{0}, \boldsymbol{a}^{0}\right)\right\rangle, \quad \Psi=\Psi\left(\boldsymbol{\varepsilon}^{0}, \boldsymbol{a}^{0}\right)$.

In the above expressions, microstrain depends on the macrostrain via the second solution Eq. (2.26) of the localization problem. In other words, the overall dissipation can be expressed too as a function of the macrostrain and the internal variables. A consequence of the assumptions Eq. (2.27) is an application of HillMandel's lemma:

$\frac{\partial \Psi}{\partial \boldsymbol{E}}=\left\langle\frac{\partial \psi^{0}\left(\boldsymbol{\varepsilon}^{0}, \boldsymbol{a}^{0}\right)}{\partial \boldsymbol{\varepsilon}^{0}}: \frac{\partial \boldsymbol{\varepsilon}^{0}}{\partial \boldsymbol{E}}\right\rangle=\left\langle\boldsymbol{\sigma}^{0}\right\rangle:\left\langle\frac{\partial \boldsymbol{\varepsilon}^{0}}{\partial \boldsymbol{E}}\right\rangle=\boldsymbol{\Sigma}$,

where we used that $\frac{\partial \varepsilon^{0}}{\partial \boldsymbol{E}}$ is an admissible strain since

$\frac{\partial \boldsymbol{\varepsilon}^{0}}{\partial \boldsymbol{E}}=\frac{\partial}{\partial \boldsymbol{E}}\left(\operatorname{sym} \nabla \boldsymbol{u}^{0}\right)=\operatorname{sym}\left(\nabla \frac{\partial \boldsymbol{u}^{0}}{\partial \boldsymbol{E}}\right)$,

$\frac{\partial \boldsymbol{u}^{0}}{\partial \boldsymbol{E}}=\boldsymbol{I} \cdot \boldsymbol{y}+\frac{\partial \boldsymbol{u}^{1}}{\partial \boldsymbol{E}}, \quad\left\langle\frac{\partial \boldsymbol{u}^{1}}{\partial \boldsymbol{E}}\right\rangle=\frac{\partial}{\partial \boldsymbol{E}}\left\langle\boldsymbol{u}^{1}\right\rangle=0$.

We note that from Eq. (2.26) the variation of microstrain is equal to $\delta \boldsymbol{\varepsilon}^{0}=\frac{\partial \boldsymbol{\varepsilon}^{0}}{\partial \boldsymbol{E}} \delta \boldsymbol{E}+\frac{\partial \boldsymbol{\varepsilon}^{0}}{\partial \boldsymbol{a}^{0}}: \delta \boldsymbol{a}^{0}$, thus

$\left\langle\boldsymbol{\sigma}^{0}: \delta \boldsymbol{\varepsilon}^{0}\right\rangle=\left\langle\boldsymbol{\sigma}^{0}\right\rangle:\left\langle\delta \boldsymbol{\varepsilon}^{0}\right\rangle=\left\langle\boldsymbol{\sigma}^{0}\right\rangle: \delta \boldsymbol{E}+\left\langle\boldsymbol{\sigma}^{0}: \frac{\partial \boldsymbol{\varepsilon}^{0}}{\partial \boldsymbol{a}^{0}}: \delta \boldsymbol{a}^{0}\right\rangle$,

therefore

$\left\langle\boldsymbol{\sigma}^{0}: \frac{\partial \varepsilon^{0}}{\partial \boldsymbol{a}^{0}}: \delta \boldsymbol{a}^{0}\right\rangle=\mathbf{0}$.

The effective generalized forces $\mathfrak{A}$ are defined in terms of the variation of the effective energy by

$\mathfrak{U}: \delta \boldsymbol{a}^{0}=-\frac{\partial \Psi}{\partial \boldsymbol{a}^{0}}: \delta \boldsymbol{a}^{0}$.

We note that this definition has a practical meaning only if the field of internal variables $\boldsymbol{a}^{0}$ is approximated by a finite number of variables according to the microstructure, a task which is not trivial.

Using the definition $\psi^{0}=\psi^{0}\left(\boldsymbol{\varepsilon}^{0}, \boldsymbol{a}^{0}\right)$ and (2.32),

$$
\begin{aligned}
-\frac{\partial \Psi}{\partial \boldsymbol{a}^{0}}: \delta \boldsymbol{a}^{0} & =-\left\langle\frac{\partial \psi^{0}\left(\boldsymbol{\varepsilon}^{0}, \boldsymbol{a}^{0}\right)}{\partial \boldsymbol{\varepsilon}^{0}}: \frac{\partial \boldsymbol{\varepsilon}^{0}}{\partial \boldsymbol{a}^{0}}: \delta \boldsymbol{a}^{0}\right\rangle-\left\langle\frac{\partial \psi^{0}\left(\boldsymbol{\varepsilon}^{0}, \boldsymbol{a}^{0}\right)}{\partial \boldsymbol{a}^{0}}: \delta \boldsymbol{a}^{0}\right\rangle \\
& =-\left\langle\frac{\partial \psi^{0}\left(\boldsymbol{\varepsilon}^{0}, \boldsymbol{a}^{0}\right)}{\partial \boldsymbol{a}^{0}}: \delta \boldsymbol{a}^{0}\right\rangle .
\end{aligned}
$$

We conclude that

$\mathfrak{U}: \delta \boldsymbol{a}^{0}=\left\langle\boldsymbol{A}: \delta \boldsymbol{a}^{0}\right\rangle$.

Based on the above, the overall dissipation $D$ is given by

$D=\langle d\rangle=\left\langle\boldsymbol{\sigma}^{0}: \dot{\boldsymbol{\varepsilon}}^{0}-\psi^{0}\right\rangle=\left\langle\boldsymbol{A}: \dot{\boldsymbol{a}}^{0}\right\rangle=\mathfrak{A}: \dot{\boldsymbol{a}}^{0}$

Therefore the homogenized dissipation inequality can be treated starting from the microstress $\sigma^{0}$ and the rates of the internal micro-hardening "forces" $\dot{\boldsymbol{q}}^{0}$ and micro-plastic strain $\dot{\boldsymbol{\varepsilon}}^{p 0}$, in correlation with the micro-yield surface $f^{0}$. This is equivalent to the statement that the micro-evolutionary equations derived from the micro-dissipation inequality satisfy the macro-dissipation inequality too.

\section{Two phase wavy multilayered composite made of elastic- perfectly plastic materials}

\subsection{Microstress in terms of macrostrain and plastic microstrains}

In this section we will present an analytical solution of the strain driven homogenization problem Eqs. (2.23)-(2.25) for a two phase wavy multilayered composite made of elastic-perfectly plastic materials. We recall that this problem admits that the macrostrain $\boldsymbol{E}$ and the internal parameters $\varepsilon^{p}$ are known in the unit cell. Generally speaking, it may be the subject of a computational method based on assumptions for the distribution of microplastic strain $\varepsilon^{p}$ (see for instance [32]. More specifically, we will present the analytical expressions for microstrain and microstress tensors,

$\boldsymbol{\varepsilon}^{0}=\boldsymbol{\varepsilon}^{0}\left(\boldsymbol{E}, \boldsymbol{\varepsilon}^{p 0}\right), \quad \boldsymbol{\sigma}^{0}=\boldsymbol{\sigma}^{0}\left(\boldsymbol{E}, \boldsymbol{\varepsilon}^{p 0}\right)$,

for the macroscopic free energy

$\Psi=\Psi\left(\boldsymbol{E}, \boldsymbol{\varepsilon}^{p 0}\right)$

the macroscopic plastic strain tensor

$\boldsymbol{E}^{p}=\boldsymbol{E}^{p}\left(\boldsymbol{E}, \boldsymbol{\varepsilon}^{p 0}\right)$

and the effective yield surface

$\Phi=\Phi\left(\boldsymbol{E}, \boldsymbol{\varepsilon}^{p 0}\right)$

In Fig. 2 we see the multilayered composite under consideration. The generalized periodicity function describing the parametric equation of the wavy surface reads $\varrho(x)=x_{2}+H \sin \left(\frac{2 \pi}{L} x_{1}\right)=$ const.

We will use the fact that in every phase of this composite, stress and strain tensors are uniform for fixed $x_{1}$ [52]. This is a result of the homogenization with respect to $x_{2}$-axis presented in Ref. [52]. However, this is a major hypothesis for a two dimensional homogenization. Due to the uniformity of stress for fixed $x_{1}$, plastic strain components are also uniform [49]. This uniformity of stress and strains allows us to follow an alternative approach from the one presented in Ref. [52]. Instead of working with a generalized periodicity function $\mathrm{o}$, one could simplify the computations by introducing the local angle $\theta$ of the layers at each point (see Fig. 2).

We recall that the microstrain is given by

$\boldsymbol{\varepsilon}^{0}(\boldsymbol{u})=\boldsymbol{E}+\boldsymbol{\varepsilon}\left(\boldsymbol{u}^{1}\right)$

with

$\left\langle\boldsymbol{\varepsilon}\left(\boldsymbol{u}^{1}\right)\right\rangle=0$.

Considering only two layers, we will use the superscripts I and II to define quantities at each phase. Under this condition, the last equation reads

$\sum_{r=I}^{I I} c^{r} \boldsymbol{\varepsilon}^{r}=\mathbf{0}$

where $c^{r}$ and $\boldsymbol{\varepsilon}^{r}(r=\mathrm{I}, \mathrm{II})$ denotes the volume fraction and the periodic part of the microstrains respectively of the phases I and II.

Considering isotropic material phases, the constitutive equation for each phase $r$ is written 
Table 1

Computational plastic algorithm.



$\boldsymbol{\sigma}^{0 r}=\boldsymbol{C}^{r}:\left(\boldsymbol{\varepsilon}^{0 r}-\boldsymbol{\varepsilon}^{p r}\right)=\boldsymbol{C}^{r}:\left(\boldsymbol{E}+\boldsymbol{\varepsilon}^{r}-\boldsymbol{\varepsilon}^{p r}\right)$,

where $\boldsymbol{C}$ denotes the isotropic elasticity tensor. Considering the local angle $\theta$ at each point of the wavy material, one could perform the usual transformation from the global $\boldsymbol{x}$ to the local $\boldsymbol{x}^{\prime}$ coordinate system

$\boldsymbol{\sigma}^{0 \prime}=\boldsymbol{Q} \cdot \boldsymbol{\sigma}^{0} \cdot \boldsymbol{Q}^{T}, \quad \boldsymbol{\varepsilon}^{\prime}=\boldsymbol{Q} \cdot \boldsymbol{\varepsilon} \cdot \boldsymbol{Q}^{T}$

where $\boldsymbol{Q}=\left(\begin{array}{ccc}\cos \theta & \sin \theta & 0 \\ -\sin \theta & \cos \theta & 0 \\ 0 & 0 & 1\end{array}\right)$.

In the sequel, we will use Voigt notation and write stress and strain tensors in the form of vectors and all the fourth order tensors as $6 \times 6$ matrices. $^{2}$ In this notation, any matrix or vector multiplication is denoted by the symbol $\{\cdot\}$ of single contraction. Using Voigt notation, continuity of tractions and strains (see Fig. 2) gives

$\sigma_{2}^{0 \prime I}=\sigma_{2}^{0 / I I}, \quad \sigma_{4}^{0 / I}=\sigma_{4}^{0 / I I}, \quad \sigma_{6}^{0 / I}=\sigma_{6}^{0 / I I}$,

and

$\varepsilon_{1}^{\prime I}=\varepsilon_{1}^{\prime I I}, \varepsilon_{3}^{\prime I}=\varepsilon_{3}^{\prime I I}, \varepsilon_{5}^{\prime I}=\varepsilon_{5}^{\prime I I}$.

From Eq. (3.7) and (3.11), it follows that $\varepsilon_{1}^{\prime r}=\varepsilon_{3}^{\prime r}=\varepsilon_{5}^{\prime r}=0, r=I, I I$. Thus, using Eq. (3.9) we obtain

$$
\begin{gathered}
\varepsilon_{1}^{r} \cos ^{2} \theta+\varepsilon_{6}^{r} \sin 2 \theta+\varepsilon_{2}^{r} \sin ^{2} \theta=0, \quad \varepsilon_{3}^{r}=0 \\
\varepsilon_{5}^{r} \cos \theta+\varepsilon_{4}^{r} \sin \theta=0
\end{gathered}
$$

for $r=$ I,II. From Eqs. (3.9) and (3.10), we obtain the following relations between the stresses:

$$
\begin{aligned}
& \left(\sigma_{1}^{O I}-\sigma_{1}^{O I I}\right) \sin ^{2} \theta-\left(\sigma_{6}^{O I}-\sigma_{6}^{O I I}\right) \sin 2 \theta+\left(\sigma_{2}^{O I}-\sigma_{2}^{O I I}\right) \cos ^{2} \theta=0, \\
& -\left(\sigma_{5}^{0 I}-\sigma_{5}^{O I I}\right) \sin \theta+\left(\sigma_{4}^{O I}-\sigma_{4}^{O I I}\right) \cos \theta=0, \\
& -\left(\sigma_{6}^{O I}-\sigma_{6}^{O I I}\right)\left(\sin ^{2} \theta-\cos ^{2} \theta\right)+\frac{1}{2}\left(\sigma_{1}^{0 I}-\sigma_{1}^{O I I}\right) \sin 2 \theta-\frac{1}{2} \sin 2 \theta\left(\sigma_{2}^{O I}-\sigma_{2}^{O I I}\right)=0 .
\end{aligned}
$$

\footnotetext{
${ }^{2}$ According to the Voigt notation, the double indices are substituted with only one, following the rule $11 \rightarrow 1,22 \rightarrow 2,33 \rightarrow 3,23 \rightarrow 4,13 \rightarrow 5,12 \rightarrow 6$. Moreover, the shear terms of the strains $\varepsilon_{4}, \varepsilon_{5}$ and $\varepsilon_{6}$ denote the shear angles $2 \varepsilon_{23}, 2 \varepsilon_{13}$ and $2 \varepsilon_{12}$ respectively.
}

Using the constitutive relations Eq. (3.8), we can rewrite Eq. (3.13) in terms of the unknown strains $\varepsilon^{I}$ and $\boldsymbol{\varepsilon}^{I I}$, 
$\left(C_{44}^{I}-C_{44}^{I I}\right) E_{6}+C_{44}^{I} \varepsilon_{6}^{I}-C_{44}^{I I} \varepsilon_{6}^{I I}-C_{44}^{I} \varepsilon_{6}^{I I}+C_{44}^{I I} \varepsilon_{6}^{p I I}=\left[\left(C_{11}^{I}-C_{11}^{I I}\right) E_{1}+\left(C_{12}^{I}-C_{12}^{I I}\right) E_{2}+\left(C_{12}^{I}-C_{12}^{I I}\right) E_{3}+C_{11}^{I} \varepsilon_{1}^{I}-C_{11}^{I I} \varepsilon_{1}^{I I}+C_{12}^{I} \varepsilon_{2}^{I}\right.$

$\left.-C_{12}^{I I} \varepsilon_{2}^{I I}+C_{12} \varepsilon_{3}^{I}-C_{12} \varepsilon_{3}^{I I}-C_{11} \varepsilon_{1}^{p I}+C_{11} \varepsilon_{1}^{p I I}-C_{12} \varepsilon_{2}^{p I}+C_{12} \varepsilon_{2}^{p I I}-C_{12} \varepsilon_{3}^{p I}+C_{12} \varepsilon_{3}^{p I I}\right] \tan \theta,\left(C_{12}^{I}-C_{12}^{I I}\right) E_{1}+\left(C_{11}^{I}-C_{11}^{I I}\right) E_{2}$

$+\left(C_{12}^{I}-C_{12}^{I I}\right) E_{3}+C_{12}^{I} \varepsilon_{1}^{I}-C_{12}^{I I} \varepsilon_{1}^{I I}+C_{11}^{I} \varepsilon_{2}^{I}-C_{11} \varepsilon_{2}^{I I}+C_{12}^{I} \varepsilon_{3}^{I}-C_{12} \varepsilon_{3}^{I I}-C_{12}^{I} \varepsilon_{1}^{p I}+C_{12} \varepsilon_{1}^{p I I}-C_{11}^{I} \varepsilon_{2}^{p I}+C_{11} \varepsilon_{2}^{p I I}-C_{12}^{I} \varepsilon_{3}^{p I}+C_{12} \varepsilon_{3}^{p I I}$

$=\left[\left(C_{44}^{I}-C_{44}^{I I}\right) E_{6}+C_{44}^{I} \varepsilon_{6}^{I}-C_{44}^{I I} \varepsilon_{6}^{I I}-C_{44}^{I} \varepsilon_{6}^{p I}+C_{44} \varepsilon_{6}^{p I I}\right] \tan \theta,\left[\left(C_{44}^{I}-C_{44}^{I I}\right) E_{4}+C_{44}^{I} \varepsilon_{4}^{I}-C_{44}^{I I} \varepsilon_{4}^{I I}-a_{44}^{I} \varepsilon_{4}^{p I}+a_{44}^{I I} \varepsilon_{4}^{p I I}\right] \tan \theta$

$=\left(C_{44}^{I}-C_{44}^{I I}\right) E_{5}+C_{44}^{I} \varepsilon_{5}^{I}-C_{44}^{I I} \varepsilon_{5}^{I I}-C_{44}^{I} \varepsilon_{5}^{p I}+C_{44}^{I I} \varepsilon_{5}^{p I I}$.

As we already mentioned in the begining of this section, the macrostrain $\boldsymbol{E}$ and the plastic strains $\varepsilon^{p I}$ and $\varepsilon^{p I I}$ are considered to be known throughout this analysis.

Using Eq. (3.7) that connects $\varepsilon_{4}^{I}$ with $\varepsilon_{4}^{I I}$, Eq. (3.12) 3 that connects $\varepsilon_{4}^{r}$ with $\varepsilon_{5}^{r}$, and Eq. (3.5), the relation Eq. (3.14) allows us to compute the total microstrains $\varepsilon_{4}^{0 I}$ and $\varepsilon_{4}^{O I I}$,

$$
\begin{aligned}
\varepsilon_{4}^{0 I}= & \left(1-\frac{c^{I I}\left(C_{44}^{I}-C_{44}^{I I}\right)}{\left(1+\tan ^{2} \theta\right)\left(c^{I I} C_{44}^{I}+c^{I} C_{44}^{I I}\right)}\right) E_{4}+c^{I I} \frac{\left(C_{44}^{I}-C_{44}^{I I}\right) \tan \theta}{\left(1+\tan ^{2} \theta\right)\left(c^{I I} C_{44}^{I}+c^{I} C_{44}^{I I}\right)} E_{5}+c^{I I} \frac{C_{44}^{I}}{\left(1+\tan ^{2} \theta\right)\left(c^{I I} C_{44}^{I}+c^{I} C_{44}^{I I}\right)} \varepsilon_{4}^{p I} \\
& -c^{I I} \frac{C_{44}^{I I}}{\left(1+\tan ^{2} \theta\right)\left(c^{I I} C_{44}^{I}+c^{I} C_{44}^{I I}\right)} \varepsilon_{4}^{p I I}-c^{I I} \frac{C_{44}^{I} \tan \theta}{\left(1+\tan ^{2} \theta\right)\left(c^{I I} C_{44}^{I}+c^{I} C_{44}^{I I}\right)} \varepsilon_{5}^{p I}+c^{I I} \frac{C_{44}^{I I} \tan \theta}{\left(1+\tan ^{2} \theta\right)\left(c^{I I} C_{44}^{I}+c^{I} C_{44}^{I I}\right)_{5}^{p I I},} \\
\varepsilon_{4}^{O I I}= & \left(1+c^{I} \frac{C_{44}^{I}-C_{44}^{I I}}{\left(1+\tan ^{2} \theta\right)\left(c^{I I} C_{44}^{I}+c^{I} C_{44}^{I I}\right)}\right) E_{4}-c^{I} \frac{\left(C_{44}^{I}-C_{44}^{I I}\right) \tan \theta}{\left(1+\tan ^{2} \theta\right)\left(c^{I I} C_{44}^{I}+c^{I} C_{44}^{I I}\right)} E_{5}-c^{I} \frac{C_{44}^{I}}{\left(1+\tan ^{2} \theta\right)\left(c^{I I} C_{44}^{I}+c^{I} C_{44}^{I I}\right)} \varepsilon_{4}^{p I} \\
& +c^{I} \frac{C_{44}^{I I}}{\left(1+\tan ^{2} \theta\right)\left(c^{I I} C_{44}^{I}+c^{I} C_{44}^{I I}\right)} \varepsilon_{4}^{p I I}+c^{I} \frac{C_{44}^{I} \tan \theta}{\left(1+\tan ^{2} \theta\right)\left(c^{I I} C_{44}^{I}+c^{I} C_{44}^{I I}\right)} \varepsilon_{5}^{p I}-c^{I} \frac{C_{44}^{I I} \tan \theta}{\left(1+\tan ^{2} \theta\right)\left(c^{I I} C_{44}^{I}+c^{I} C_{44}^{I I}\right)} \varepsilon_{5}^{p I I} .
\end{aligned}
$$

The connection between $\varepsilon_{4}^{r}$ with $\varepsilon_{5}^{r}$ through Eq. (3.12) 3 and the main Eq. (3.5), allow us to use the relations Eq. (3.15) and evaluate also $\varepsilon_{5}^{\mathrm{OI}}$ and $\varepsilon_{5}^{\mathrm{OII}}$.

Solving Eq. (3.12) $)_{1}$ with regard to $\varepsilon_{1}^{I}$, using Eq. (3.7) and substituting in Eq. (3.14) $)_{1}$ and Eq. (3.14) 2 , we obtain

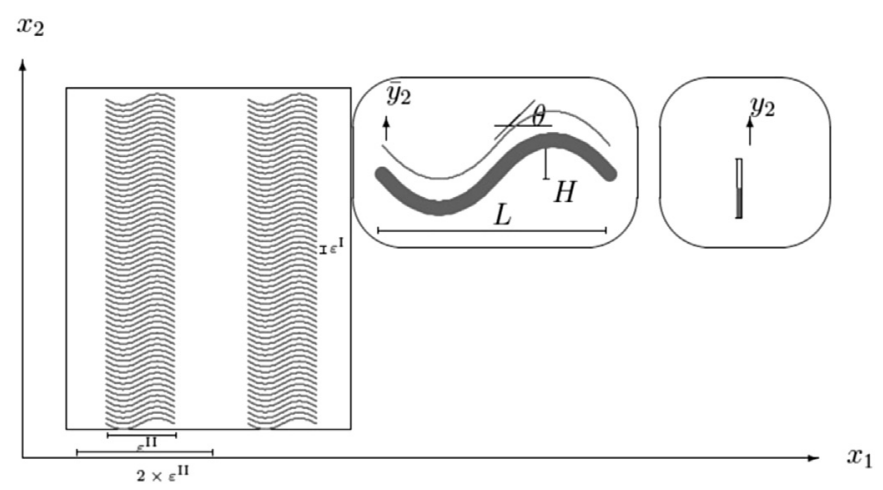

Fig. 3. Laminate reinforced composite periodic structure with wavy inclusions and the unit cell in the framework of generalized periodicity function in micro-coordinate system.

$$
\begin{aligned}
& {\left[\left(C_{44}^{I}+\frac{c^{I}}{c^{I I}} C_{44}^{I I}\right)+2 \tan ^{2} \theta\left(C_{11}^{I}+\frac{c^{I}}{c^{I I}} C_{11}^{I I}\right)\right] \varepsilon_{6}^{I}} \\
& +\left[\left(C_{11}^{I}+\frac{c^{I}}{c^{I I}} C_{11}^{I I}\right) \tan ^{3} \theta-\left(C_{12}^{I}+\frac{c^{I}}{c^{I I}} C_{12}^{I I}\right) \tan \theta\right] \varepsilon_{2}^{I} \\
& =\left(C_{11}^{I}-C_{11}^{I I}\right) E_{1} \tan \theta+\left(C_{12}^{I}-C_{12}^{I I}\right) E_{2} \tan \theta \\
& +\left(C_{12}^{I}-C_{12}^{I I}\right) E_{3} \tan \theta-\left(C_{44}^{I}-C_{44}^{I I}\right) E_{6}-C_{11}^{I} \varepsilon_{1}^{p I} \tan \theta \\
& +C_{11}^{I I} \varepsilon_{1}^{p I I} \tan \theta-C_{12}^{I} \varepsilon_{2}^{p I} \tan \theta+C_{12}^{I I} \varepsilon_{2}^{p I I} \tan \theta-C_{12}^{I} \varepsilon_{3}^{p I} \tan \theta \\
& +C_{12}^{I I} \varepsilon_{3}^{\text {pII }} \tan \theta+C_{44}^{I} \varepsilon_{6}^{\text {pI }}-C_{44}^{I I} \varepsilon_{6}^{\text {pII }} \text {, }
\end{aligned}
$$

and

Table 2

Properties of constituents.

\begin{tabular}{llc}
\hline Property & Elastic (ceramic) & Elastoplastic (metal) \\
\hline Young Modulus (GPa) & 420 & 72.40 \\
Poisson Ratio & 0.25 & 0.33 \\
Yield Stress (GPa) & - & 0.28667 \\
Volume fraction & $10 \%$ & $90 \%$ \\
\hline
\end{tabular}


$\left[2\left(C_{12}^{I}+\frac{c^{I}}{c^{I I}} C_{12}^{I I}\right)+\left(C_{44}^{I}+\frac{c^{I}}{c^{I I}} C_{44}^{I I}\right)\right] \varepsilon_{6}^{I} \tan \theta+\left[\left(C_{12}^{I}+\frac{c^{I}}{c^{I I}} C_{12}^{I I}\right) \tan ^{2} \theta\right.$
$\left.-\left(C_{11}^{I}+\frac{c^{I}}{c^{I I}} C_{11}^{I I}\right)\right] \varepsilon_{2}^{I}=\left(C_{12}^{I}-C_{12}^{I I}\right) E_{1}+\left(C_{11}^{I}-C_{11}^{I I}\right) E_{2}$
$+\left(C_{12}^{I}-C_{12}^{I I}\right) E_{3}-\left(C_{44}^{I}-C_{44}^{I I}\right) E_{6} \tan \theta-C_{12}^{I} \varepsilon_{1}^{p I}+C_{12}^{I I} \varepsilon_{1}^{p I I}$
$-C_{11}^{I} \varepsilon_{2}^{p I}+C_{11}^{I I} \varepsilon_{2}^{p I I}-C_{12}^{I} \varepsilon_{3}^{p I}+C_{12}^{I I} \varepsilon_{3}^{p I I}+C_{44}^{I} \varepsilon_{6}^{p I} \tan \theta-C_{44}^{I I} \varepsilon_{6}^{p I I} \tan \theta$

respectively. Similarly, solving Eq. (3.12) 1 with regard to $\varepsilon_{1}^{I I}$, using Eq. (3.7) and substituting in (3.14) 1 and (3.14) 2 , we obtain

$$
\begin{aligned}
& {\left[\left(C_{44}^{I I}+\frac{c^{I I}}{c^{I}} C_{44}^{I}\right)+2 \tan ^{2} \theta\left(C_{11}^{I I}+\frac{c^{I I}}{c^{I}} C_{11}^{I}\right)\right] \varepsilon_{6}^{I I}} \\
& +\left[\left(C_{11}^{I I}+\frac{c^{I I}}{c^{I}} C_{11}^{I}\right) \tan ^{3} \theta-\left(C_{12}^{I I}+\frac{c^{I I}}{c^{I}} C_{12}^{I}\right) \tan \theta\right] \varepsilon_{2}^{I I} \\
& =-\left(C_{11}^{I}-C_{11}^{I I}\right) E_{1} \tan \theta-\left(C_{12}^{I}-C_{12}^{I I}\right) E_{2} \tan \theta \\
& -\left(C_{12}^{I}-C_{12}^{I I}\right) E_{3} \tan \theta+\left(C_{44}^{I}-C_{44}^{I I}\right) E_{6}+C_{11}^{I} \varepsilon_{1}^{p I} \tan \theta \\
& -C_{11}^{I I} \varepsilon_{1}^{p I I} \tan \theta+C_{12}^{I} \varepsilon_{2}^{p I} \tan \theta-C_{12}^{I I} \varepsilon_{22}^{p I I} \tan \theta+C_{12}^{I} \varepsilon_{3}^{p I} \tan \theta \\
& -C_{12}^{I I} \varepsilon_{3}^{p I I} \tan \theta-C_{44}^{I} \varepsilon_{6}^{p I}+C_{44}^{I I} \varepsilon_{6}^{p I I},
\end{aligned}
$$

and

$$
\begin{aligned}
& {\left[2\left(C_{12}^{I I}+\frac{c^{I I}}{c^{I}} C_{12}^{I}\right)+\left(C_{44}^{I I}+\frac{c^{I I}}{c^{I}} C_{44}^{I}\right)\right] \varepsilon_{6}^{I I} \tan \theta} \\
& +\left[\left(C_{12}^{I I}+\frac{c^{I I}}{c^{I}} C_{12}^{I}\right) \tan ^{2} \theta-\left(C_{11}^{I I}+\frac{c^{I I}}{c^{I}} C_{11}^{I}\right)\right] \varepsilon_{2}^{I I} \\
& =-\left(C_{12}^{I}-C_{12}^{I I}\right) E_{1}-\left(C_{11}^{I}-C_{11}^{I I}\right) E_{2}-\left(C_{12}^{I}-C_{12}^{I I}\right) E_{3} \\
& +\left(C_{44}^{I}-C_{44}^{I I}\right) E_{6} \tan \theta+C_{12}^{I} \varepsilon_{1}^{p I}-C_{12}^{I I} \varepsilon_{1}^{p I I}+C_{11}^{I} \varepsilon_{2}^{p I} \\
& -C_{11}^{I I} \varepsilon_{2}^{p I I}+C_{12}^{I} \varepsilon_{3}^{p I}-C_{12}^{I I} \varepsilon_{3}^{p I I}-C_{44}^{I} \varepsilon_{6}^{p I} \tan \theta+C_{44}^{I I} \varepsilon_{6}^{p I I} \tan \theta
\end{aligned}
$$

respectively. The system of Eqs (3.16)-(3.19) for the unknowns $\varepsilon_{2}^{r}$ and $\varepsilon_{6}^{r}(r=1,2)$ takes the linear form

$$
\boldsymbol{M}^{r} \cdot \chi^{r}=\boldsymbol{N}^{r}
$$

where $\boldsymbol{\chi}^{r}=\left(\begin{array}{ll}\varepsilon_{2}^{r} & \varepsilon_{6}^{r}\end{array}\right)^{T}$ and the matrices $\boldsymbol{M}^{r}$ and $\boldsymbol{N}^{r}$ are given in Appendix A for $r=I$.

Finally, the microstrains, in compact form are given by

$\boldsymbol{\varepsilon}^{0 r}=\boldsymbol{G}_{E}^{r} \cdot \boldsymbol{E}+\sum_{B=I}^{I I} \boldsymbol{G}_{B}^{r} \cdot \boldsymbol{\varepsilon}^{p B}$,

where $\boldsymbol{G}_{E}^{r}, \boldsymbol{G}_{I}^{r}$ and $\boldsymbol{G}_{I I}^{r}$ are $6 \times 6$ matrices, whose components are given in Appendices B and C. The quantities $\boldsymbol{G}_{E}^{r}$ represent the elastic strain concentration tensors [44].

Moreover, the microstresses are given by Eq. (3.8). These expressions, after proper manipulations are written

$\boldsymbol{\sigma}^{0 r}=\boldsymbol{C}^{r} \cdot \boldsymbol{G}_{E}^{r} \cdot \boldsymbol{E}-\sum_{B=I}^{I I} \boldsymbol{S}_{B}^{r} \cdot \boldsymbol{\varepsilon}^{p B}$,

where
$\boldsymbol{S}_{I}^{I}=\boldsymbol{C}^{I} \cdot\left(\boldsymbol{I}-\boldsymbol{G}_{I}^{I}\right), \quad \boldsymbol{S}_{I I}^{I}=-\boldsymbol{C}^{I} \cdot \boldsymbol{G}_{I I}^{I}$

$\boldsymbol{S}_{I}^{I I}=-\boldsymbol{C}^{I I} \cdot \boldsymbol{G}_{I}^{I I}, \quad \boldsymbol{S}_{I I}^{I I}=\boldsymbol{C}^{I I} \cdot\left(\boldsymbol{I}-\boldsymbol{G}_{I I}^{I I}\right)$,

and $I$ is the $6 \times 6$ identity matrix. The two last terms in the expression of microstress can be viewed as initial stress vectors in the computational process (see Ref. [12]. It is worth noting that all matrices $\boldsymbol{G}_{E}^{I, I I}$ and $\boldsymbol{G}_{I, I I}^{I, I I}$ depend on $\tan \theta$.

\subsection{Effective constitutive law}

In order to compute the macrostress $\boldsymbol{\Sigma}$ in terms of the internal variables, we need the macro-free energy, which is equal to the average of the corresponding microenergy, namely

$W^{e l}=\frac{1}{2}\left\langle\sigma^{0} \cdot \varepsilon^{e l}\right\rangle$,

and through Eq. (3.22)

$$
\begin{aligned}
W^{e l}= & \frac{1}{2} c^{I}\left(\boldsymbol{C}^{I} \cdot \boldsymbol{G}_{E}^{I} \cdot \boldsymbol{E}+\boldsymbol{C}^{I} \cdot\left(\boldsymbol{G}_{I}^{I}-\boldsymbol{I}\right) \cdot \boldsymbol{\varepsilon}^{p I}\right) \cdot\left(\boldsymbol{E}-\boldsymbol{\varepsilon}^{p I}\right) \\
& +\frac{1}{2} c^{I}\left(\boldsymbol{C}^{I} \cdot \boldsymbol{G}_{I I}^{I} \cdot \boldsymbol{\varepsilon}^{p I I}\right) \cdot\left(\boldsymbol{E}-\boldsymbol{\varepsilon}^{p I}\right)+\frac{1}{2} c^{I I}\left(\boldsymbol{C}^{I I} \cdot \boldsymbol{G}_{E}^{I I} \cdot \boldsymbol{E}+\boldsymbol{C}^{I I} \cdot \boldsymbol{G}_{I}^{I I} \cdot \boldsymbol{\varepsilon}^{p I}\right) \\
& \times\left(\boldsymbol{E}-\boldsymbol{\varepsilon}^{p I I}\right)+\frac{1}{2} c^{I I}\left(\boldsymbol{C}^{I I} \cdot\left(\boldsymbol{G}_{I I}^{I I}-\boldsymbol{I}\right) \cdot \boldsymbol{\varepsilon}^{p I I}\right) \cdot\left(\boldsymbol{E}-\boldsymbol{\varepsilon}^{p I I}\right) .
\end{aligned}
$$

Macrostress, through Eq. (3.25) can be computed as follows,

$$
\begin{aligned}
\boldsymbol{\Sigma}= & \frac{\partial W^{e l}}{\partial \boldsymbol{E}}=\left(c^{I} \boldsymbol{C}^{I} \cdot \boldsymbol{G}_{E}^{I}+c^{I I} \boldsymbol{C}^{I I} \cdot \boldsymbol{G}_{E}^{I I}\right) \cdot \boldsymbol{E}+\frac{1}{2}\left(c^{I} \boldsymbol{C}^{I} \cdot\left(\boldsymbol{G}_{I}^{I}-\boldsymbol{I}\right)-c^{I} \boldsymbol{C}^{I} \cdot \boldsymbol{G}_{E}^{I}\right. \\
& \left.+c^{I I} \boldsymbol{C}^{I I} \cdot \boldsymbol{G}_{I}^{I I}\right) \cdot \boldsymbol{\varepsilon}^{p I}+\frac{1}{2}\left(c^{I I} \boldsymbol{C}^{I I} \cdot\left(\boldsymbol{G}_{I I}^{I I}-\boldsymbol{I}\right)-c^{I I} \boldsymbol{C}^{I I} \cdot \boldsymbol{G}_{E}^{I I}+c^{I} \boldsymbol{C}^{I} \cdot \boldsymbol{G}_{I I}^{I}\right) \cdot \boldsymbol{\varepsilon}^{p I I}
\end{aligned}
$$

Setting

$\boldsymbol{C}^{h o m}=\sum_{r=I}^{I I} c^{r} \boldsymbol{C}^{r} \cdot \boldsymbol{G}_{E}^{r}$,

and

$$
\begin{aligned}
& \boldsymbol{R}^{I}=\frac{1}{2}\left(\boldsymbol{C}^{h o m}\right)^{-1} \cdot\left(c^{I} \boldsymbol{C}^{I} \cdot\left(\boldsymbol{G}_{I}^{I}-\boldsymbol{I}\right)-c^{I} \boldsymbol{C}^{I} \cdot \boldsymbol{G}_{E}^{I}+c^{I I} \boldsymbol{C}^{I I} \cdot \boldsymbol{G}_{I}^{I I}\right) \\
& \boldsymbol{R}^{I I}=\frac{1}{2}\left(\boldsymbol{C}^{h o m}\right)^{-1} \cdot\left(c^{I I} \boldsymbol{C}^{I I} \cdot\left(\boldsymbol{G}_{I I}^{I I}-\boldsymbol{I}\right)-c^{I I} \boldsymbol{C}^{I I} \cdot \boldsymbol{G}_{E}^{I I}+c^{I} \boldsymbol{C}^{I} \cdot \boldsymbol{G}_{I I}^{I}\right) \\
& \boldsymbol{E}^{p}=\sum_{r=I}^{I I} \boldsymbol{R}^{r} \cdot \boldsymbol{\varepsilon}^{p r}
\end{aligned}
$$

we have

$\boldsymbol{\Sigma}=\boldsymbol{C}^{\text {hom }} \cdot\left(\boldsymbol{E}-\boldsymbol{E}^{p}\right)$.

By introducing the quantities for the $r$ phase

$\boldsymbol{H}_{\Sigma}^{r}=\boldsymbol{C}^{r} \cdot \boldsymbol{G}_{E}^{r} \cdot\left(\boldsymbol{C}^{h o m}\right)^{-1}$

$\boldsymbol{\sigma}_{\text {res }}^{0 r}=\boldsymbol{C}^{r} \cdot \boldsymbol{G}_{E}^{r} \cdot \boldsymbol{E}^{p}-\sum_{B=I}^{I I} \boldsymbol{S}_{B}^{r} \cdot \boldsymbol{\varepsilon}^{p B}$,

and using Eq. (3.22) and Eq. (3.29), microstresses can be written as 


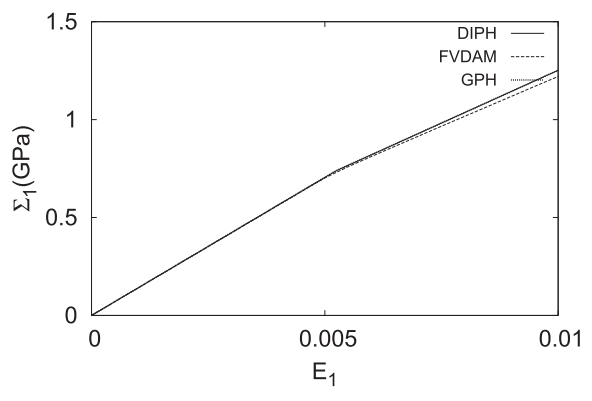

(a)

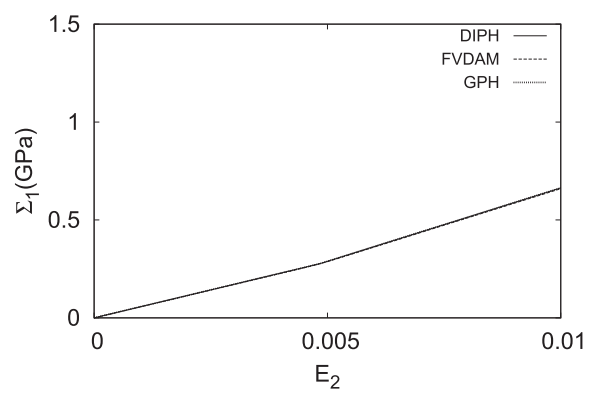

(c)

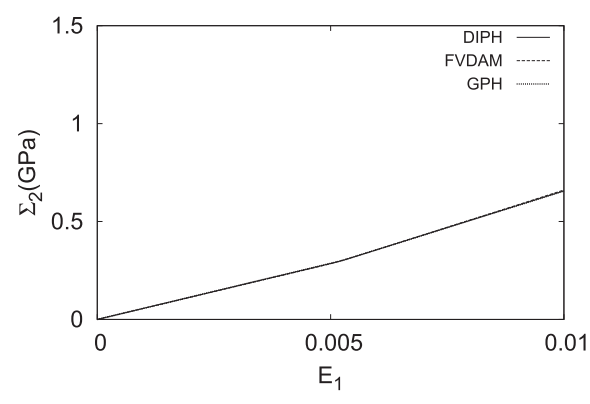

(b)

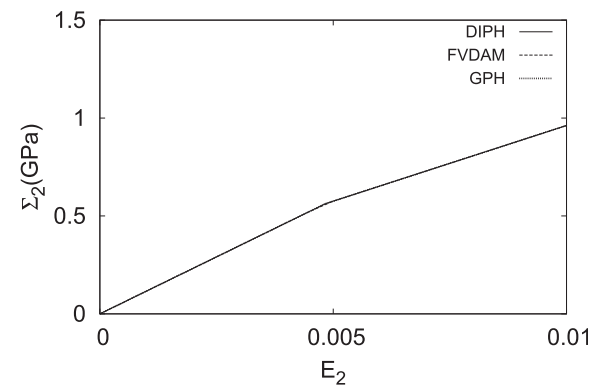

(d)

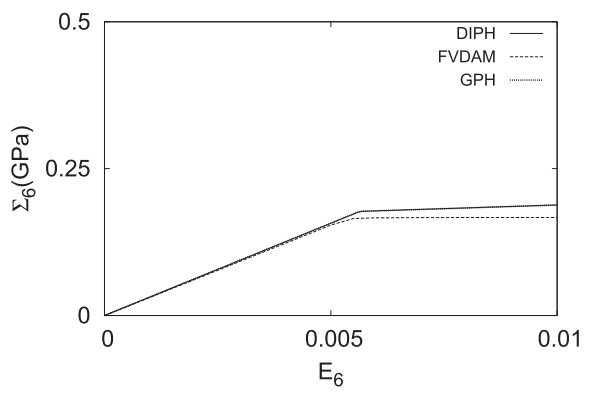

(e)

Fig. 4. Macrostress vs macrostrain for waviness 0.05 and strain increment $\Delta E_{1}$ (a and b), $\Delta E_{2}$ (c and d) and $\Delta E_{6}$ (e).

$\boldsymbol{\sigma}^{0 r}=\boldsymbol{H}_{\Sigma}^{r} \cdot \boldsymbol{\Sigma}+\boldsymbol{\sigma}_{\text {res }}^{0 r}$

where $\boldsymbol{H}_{\Sigma}^{I}$ and $\boldsymbol{H}_{\Sigma}^{I I}$ are the elastic stress concentration tensors [44] and $\boldsymbol{\sigma}_{\text {res }}^{\mathrm{OI}}$ and $\boldsymbol{\sigma}_{\text {res }}^{\text {OII }}$ are the residual stress tensors.

The computational plastic algorithm of the proposed method is presented in Table 1.

\subsection{Macroscopic yield surface}

Following Suquet [49], we first derive the dual macroscopic potential as the average of the corresponding microscopic

$\Phi^{*}=\left\langle\phi^{*}\left(\boldsymbol{\sigma}^{0}\right)\right\rangle$,

where $\phi^{* I}$ and $\phi^{* I I}$ are the indicators of the yield surfaces of the $I$ and II constituents respectively
$d^{I, I I}=\left(\boldsymbol{\sigma}, f\left(\boldsymbol{\sigma}^{i}\right)=0\right)$

where $f$ is the yield function. Then the macroscopic yield surface is given by

$D=\left[\Sigma, \Phi^{*}=0\right]$.

Since

$\Phi^{* I, I I}=0$

the macroscopic yield surface is the internal envelope of $d^{I}$ and $d^{I I}$ and has the expression

$D=\left(\boldsymbol{\Sigma}, f^{I, I I}\left(\boldsymbol{H}_{\Sigma}^{I, I I} \cdot \boldsymbol{\Sigma}+\boldsymbol{\sigma}_{\text {res }}^{0 I, I I}\right)\right)$

The initial yield surface $\left(\boldsymbol{\sigma}_{\text {res }}^{\text {OI,II }}=0\right)$ is given by 
$D^{\text {init }}=\left(\boldsymbol{\Sigma}, F^{I, I I}(\boldsymbol{\Sigma})=0\right)$

$F^{I, I I}(\boldsymbol{\Sigma})=f\left(\boldsymbol{H}_{\Sigma}^{I, I I} \cdot \boldsymbol{\Sigma}\right)$.

The macroscopic yield criterion is given by

$F=c^{I} f^{I}+c^{I I} f^{I I}$

The von Mises yield criterion for an isotropic component $r$ in the principal stress space can be written as follows,

$\left(\left(\sigma_{1}^{r}-\sigma_{2}^{r}\right)^{2}+\left(\sigma_{2}^{r}-\sigma_{3}^{r}\right)^{2}+\left(\sigma_{3}^{r}-\sigma_{1}^{r}\right)^{2}\right)^{\frac{1}{2}}=\sqrt{2} \sigma_{Y}^{r}$,

where $r=$ I,II. Through Eqs. (3.32), (3.39) and (3.40) we have the yield criterion for the composite as follows, case of elastic isotropic constituents. The first homogenization step with respect to direction $x_{2}$ leads to a continuously graded, in Ref. $x_{1}$ direction, material. The second homogenization step with respect to $x_{1}$-direction, leads to an orthotropic effective material. In this section, we will extend this method to the case of two elasticperfectly plastic constituents. We note that for the composite of Fig. 3 the continuously graded material obtained from the first step of homogenization includes the layers of the matrix perpendicular to $x_{1}$.

We present the example of a wavy layered two-phase composite structure with waviness equal to $0.05,0.20$ and 0.40 . The waviness is defined as the ratio of the amplitude $H$ to the wavelength $L, \frac{H}{L}$ (see Fig. 3). The composite is made of an elastic and an elastic-perfectly plastic material, whose properties are shown in Table 2 . We use the algorithm presented in Section 3.2 (for convenience we use the

$$
\sum_{r=I}^{I I} c^{r}\left(\Sigma_{1}^{2} A_{1}^{r}+\Sigma_{2}^{2} A_{2}^{r}+\Sigma_{3}^{2} A_{3}^{r}+\Sigma_{1} \Sigma_{2} A_{4}^{r}+\Sigma_{2} \Sigma_{3} A_{5}^{r}+\Sigma_{3} \Sigma_{1} A_{6}^{r}+\Sigma_{1} A_{7}^{r}+\Sigma_{2} A_{8}^{r}+\Sigma_{3} A_{9}^{r}+A_{10}^{r}\right)^{\frac{1}{2}}-\sum_{r=I}^{I I} c^{r} \sqrt{2} \sigma_{Y}^{r}=0
$$

where $A_{i}^{r}\left(\boldsymbol{H}_{\Sigma}^{r}\right)$ for $i=1,2,3,4,5,6$ material parameters and $A_{i}^{r}\left(\boldsymbol{H}_{\Sigma}^{r}, \boldsymbol{\sigma}_{\text {res }}^{r}\right)$ for $i=7,8,9,10$ depend on the stress concentration tensor $\boldsymbol{H}_{\Sigma}$ and the strain-history-dependent residual microstresses $\boldsymbol{\sigma}_{\text {res }}^{r}$, given by Eq. (3.31), abbreviation DIPH: Dissipation Inequality-based Periodic Homogenization) and we compare with the other two methods, GPH: Generalized Periodicity Homogenization [52] and FVDAM: Finite Volume Direct Averaging Method.

$$
\begin{aligned}
& A_{1}^{r}=\left(H_{\Sigma 11}^{r}-H_{\Sigma 21}^{r}\right)^{2}+\left(H_{\Sigma 21}^{r}-H_{\Sigma 31}^{r}\right)^{2}+\left(H_{\Sigma 31}^{r}-H_{\Sigma 11}^{r}\right)^{2} \\
& A_{2}^{r}=\left(H_{\Sigma 12}^{r}-H_{\Sigma 22}^{r}\right)^{2}+\left(H_{\Sigma 22}^{r}-H_{\Sigma 32}^{r}\right)^{2}+\left(H_{\Sigma 32}^{r}-H_{\Sigma 12}^{r}\right)^{2} \text {, } \\
& A_{3}^{r}=\left(H_{\Sigma 13}^{r}-H_{\Sigma 23}^{r}\right)^{2}+\left(H_{\Sigma 23}^{r}-H_{\Sigma 33}^{r}\right)^{2}+\left(H_{\Sigma 33}^{r}-H_{\Sigma 13}^{r}\right)^{2} \text {, } \\
& A_{4}^{r}=2\left(H_{\Sigma 11}^{r}-H_{\Sigma 21}^{r}\right)\left(H_{\Sigma 12}^{r}-H_{\Sigma 22}^{r}\right)+2\left(H_{\Sigma 21}^{r}-H_{\Sigma 31}^{r}\right)\left(H_{\Sigma 22}^{r}-H_{\Sigma 32}^{r}\right)+2\left(H_{\Sigma 31}^{r}-H_{\Sigma 11}^{r}\right)\left(H_{\Sigma 32}^{r}-H_{\Sigma 12}^{r}\right) \text {, } \\
& A_{5}^{r}=2\left(H_{\Sigma 12}^{r}-H_{\Sigma 22}^{r}\right)\left(H_{\Sigma 13}^{r}-H_{\Sigma 23}^{r}\right)+2\left(H_{\Sigma 22}^{r}-H_{\Sigma 32}^{r}\right)\left(H_{\Sigma 23}^{r}-H_{\Sigma 33}^{r}\right)+2\left(H_{\Sigma 32}^{r}-H_{\Sigma 12}^{r}\right)\left(H_{\Sigma 33}^{r}-H_{\Sigma 13}^{r}\right) \text {, } \\
& A_{6}^{r}=2\left(H_{\Sigma 11}^{r}-H_{\Sigma 21}^{r}\right)\left(H_{\Sigma 13}^{r}-H_{\Sigma 23}^{r}\right)+2\left(H_{\Sigma 21}^{r}-H_{\Sigma 31}^{r}\right)\left(H_{\Sigma 23}^{r}-H_{\Sigma 33}^{r}\right)+2\left(H_{\Sigma 31}^{r}-H_{\Sigma 11}^{r}\right)\left(H_{\Sigma 33}^{r}-H_{\Sigma 13}^{r}\right) \text {, } \\
& A_{7}^{r}=2\left(H_{\Sigma 11}^{r}-H_{\Sigma 21}^{r}\right)\left(\sigma_{\text {res } 1}^{r}-\sigma_{\text {res } 2}^{r}\right)+2\left(H_{\Sigma 21}^{r}-H_{\Sigma 31}^{r}\right)\left(\sigma_{\text {res } 2}^{r}-\sigma_{\text {res } 3}^{r}\right)+2\left(H_{\Sigma 31}^{r}-H_{\Sigma 11}^{r}\right)\left(\sigma_{\text {res } 3}^{r}-\sigma_{\text {res } 1}^{r}\right) \text {, } \\
& A_{8}^{r}=2\left(H_{\Sigma 12}^{r}-H_{\Sigma 22}^{r}\right)\left(\sigma_{\text {res } 1}^{r}-\sigma_{\text {res } 2}^{r}\right)+2\left(H_{\Sigma 22}^{r}-H_{\Sigma 32}^{r}\right)\left(\sigma_{\text {res } 2}^{r}-\sigma_{\text {res } 3}^{r}\right)+2\left(H_{\Sigma 32}^{r}-H_{\Sigma 12}^{r}\right)\left(\sigma_{\text {res } 3}^{r}-\sigma_{\text {res } 1}^{r}\right) \text {, } \\
& A_{9}^{r}=2\left(H_{\Sigma 13}^{r}-H_{\Sigma 23}^{r}\right)\left(\sigma_{\text {res } 1}^{r}-\sigma_{\text {res } 2}^{r}\right)+2\left(H_{\Sigma 23}^{r}-H_{\Sigma 33}^{r}\right)\left(\sigma_{\text {res } 2}^{r}-\sigma_{\text {res } 3}^{r}\right)+2\left(H_{\Sigma 33}^{r}-H_{\Sigma 13}^{r}\right)\left(\sigma_{\text {res } 3}^{r}-\sigma_{\text {res } 1}^{r}\right) \text {, } \\
& A_{10}^{r}=\left(\sigma_{\text {res } 1}^{r}-\sigma_{\text {res } 2}^{r}\right)^{2}+\left(\sigma_{\text {res } 2}^{r}-\sigma_{\text {res } 3}^{r}\right)^{2}+\left(\sigma_{\text {res } 3}^{r}-\sigma_{\text {res } 1}^{r}\right)^{2} \text {. }
\end{aligned}
$$

Coefficients $A_{7}, A_{8}, A_{9}, A_{10}$ of the macroscopic yield criterion Eq (3.42), which depend on residual microstresses, act as hardening parameters.

In conclusion, in this section, we presented the analytical homogenization following direction $x_{2}$ of a wavy multilayer under the assumption that the distance between the wavy layers is very small compared to the wavelength $L$ (see Fig. 2). The result of this homogenization is a continuously graded (following direction $x_{1}$ ) effective material. In the next section we will present an approximate two-steps homogenization of a locally periodic composite.

\section{Approximate two-steps homogenization of locally periodic composites with wavy inclusions}

In this section, we investigate the case of a composite consisting of a matrix with periodically ordered wavy inclusions (see Fig. 3). In Ref. [53] a two-steps homogenization scheme is presented for the
The numerical results are obtained from a MATLAB code that we have developed in order to solve the algorithm of Section 3.2. The input data for this new approach are macrostrain and micro-plastic strains. We investigate the response of the unit cell to three strain histories, corresponding to normal macrostrains $E_{1}, E_{2}$ and shear macrostrain $E_{6}$ respectively, starting from a deformation-free state up to a maximun value of macrostrain 0.01 (all the other constituents of macrostrain equal to zero). We apply the computation procedure to 21 discrete points on $x_{1}$ direction, having different values of $\theta$, and obtain effective values of stress and strain at every discrete point. Next, we apply the trapezoidal rule over entire length $L$ in order to obtain the mean values of effective stress and strain at the macropoint.

In Figs. 4-6 effective stresses $\Sigma_{1}$ and $\Sigma_{2}$ are depicted with respect to the corresponding effective strains for the three methods (DIPH, GPH and FVDAM) for waviness values 0.05, 


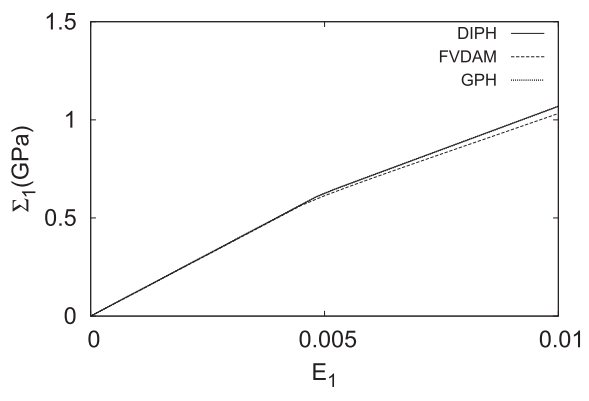

(a)

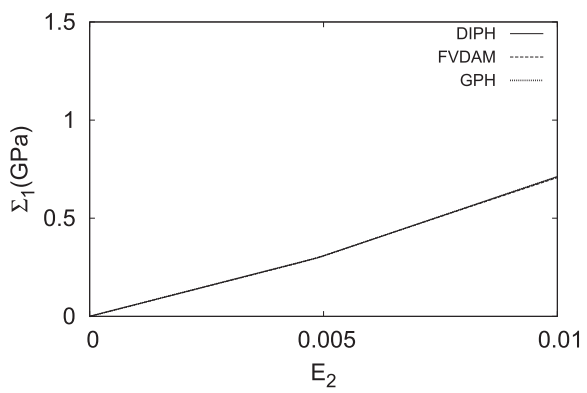

(c)

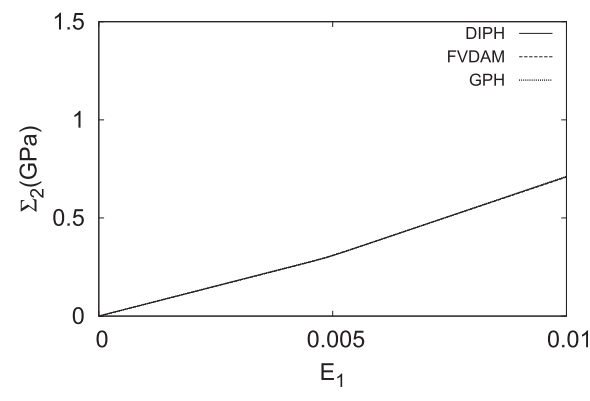

(b)

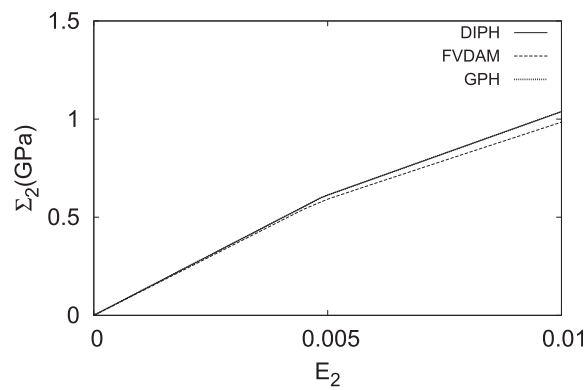

(d)

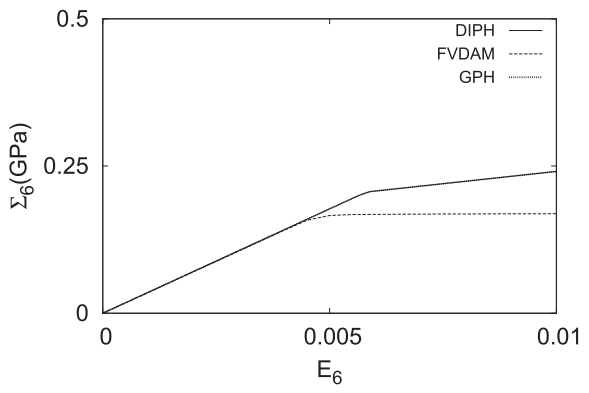

(e)

Fig. 5. Macrostress vs macrostrain for waviness 0.20 and strain increment $\Delta E_{1}$ (a and b), $\Delta E_{2}$ (c and d) and $\Delta E_{6}$ (e).

0.20 and 0.40 . We observe a total coincidence between DIPH and GPH. In addition, a total coincidence between these two methods and FVDAM for flat structures exists. On the contrary, a maximun difference $10 \%$ to effective stresses between DIPH and GPH on the one hand, and FVDAM on the other hand, is observed for waviness values $0.05,0.20$ and 0.40 . A reason for the difference is that in Refs. DIPH and GPH, homogenization is performed in two successive steps. In general, all three methods show similar behavior to the same boundary conditions. Moreover, under pure shear loading $E_{6}$, in $D I P H$ and $G P H$ waviness causes a more important hardening than in FVDAM. In other words, the elastic ("stiff") constituent is able to support additional load. This hardening effect is not monotone as waviness increases (see Figs. 4(e), 5(e) and 6 (e)). In addition, yielding occurs in a higher value of stress and strain in comparison with the flat structure. DIPH and GPH overestimate $\Sigma_{6}$ compared to FVDAM. In the case of a flat structure, elastic-perfect plastic material dominates to the effective behavior since, due to continuity conditions Eq. (3.10) and through constitutive law, an unilateral behavior is expected with the elastic ("stiff") constituent not being able to support additional load. This is not observed in the case of a wavy structure, where the elastic ("stiff") material contribute to the "burden sharing".

In order to compute the macroscopic yield surface for three given sets of macrostrain and residual microstresses, we use Wolfram Mathematica 7.0 to represent Eq. (3.41). The first set comprises macrostrain $E_{1}=0.004$ and residual microstresses equal to 0 (initial yield surface), the second set comprises macrostrain $E_{1}=0.01$ and the corresponding residual microstresses, and the third set comprises macrostrain $E_{1}=0.04$ and the corresponding residual microstresses, obtained in a wavy structure (waviness 0.40 ) with the properties of Table 1. The values of parameters $A_{i}$ are computed for the straining cases under study from Eq. (3.42) by applying the trapezoidal rule to the results given by the algorithm in discrete points. The shape of the yield surface depends on the applied boundary conditions. From the comparison of the yield surfaces, depicted in Fig. 7, a slight displacement of the yield surface can be observed.

Finally, cyclic loading is studied in the case of a flat and a wavy structure (waviness 0.40). Equal increments of macrostrain 


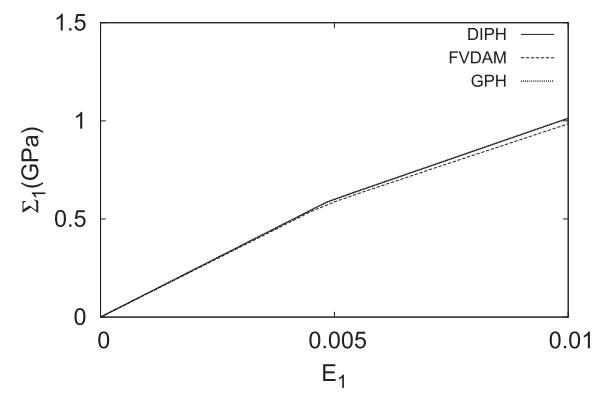

(a)

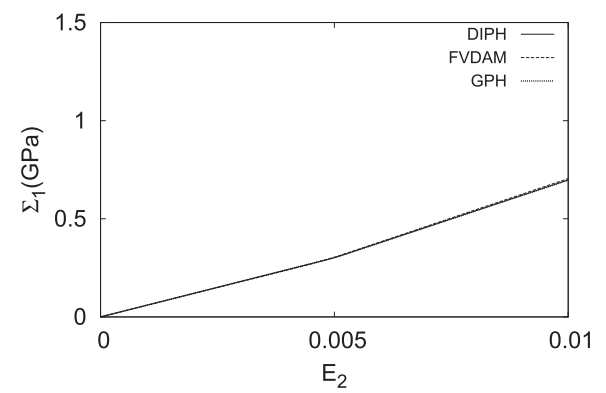

(c)

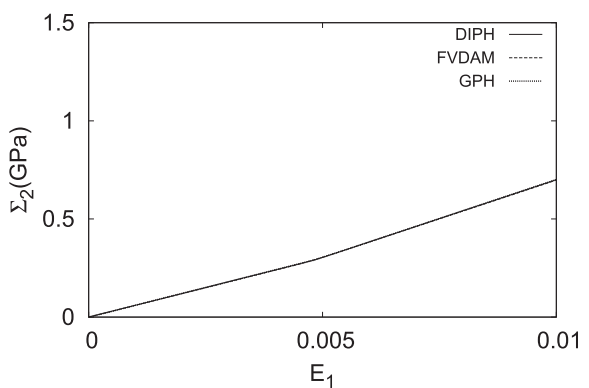

(b)

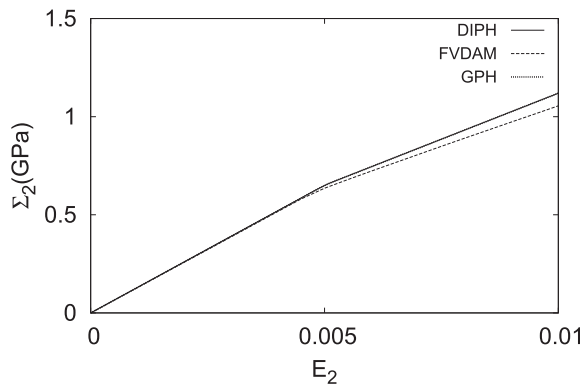

(d)

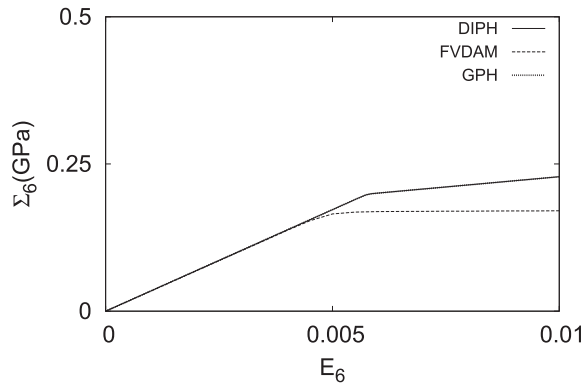

(e)

Fig. 6. Macrostress vs macrostrain for waviness 0.40 and strain increment $\Delta E_{1}$ (a and b), $\Delta E_{2}$ (c and d) and $\Delta E_{6}$ (e).

along $x_{2}$ axis are applied. A stabilization appears without kinematic hardening (see Fig. 8). We note that we also performed numerical examples for a composite made of bilinear elastic-plastic constituents and a kinematic hardening effective behavior is observed. This result, as well as additional results concerning the effective behavior of locally periodic composites with wavy inclusions, will be presented in a forthcoming paper.

It could be of interest to note that the proposed method differs from the method described in Ref. [52] in the following points: ( $i$ ) all functions are now expressed in terms of $\boldsymbol{E}$ and $\boldsymbol{\varepsilon}^{\boldsymbol{p}}$, (ii) the proposed procedure needs only the computation of the effective elastic modulus $\boldsymbol{C}^{\text {hom }}$ instead of the effective tangent modulus $\boldsymbol{D}^{\text {eff }}$ with computation saving.

\section{Conclusions}

In this paper, the analytical homogenization of wavy multilayered media made of elastic-perfectly plastic constituents is presented. Based on the generalized periodicity of stress and strains, all micro-variables are expressed in terms of the macrostrain and the microplastic strains, which are the internal variables in the cell. Assuming additivity of dissipation and energy, the macroconstitutive law is then derived from the overall energy function and dual dissipation potential. Moreover, analytical expressions for the macroelasticity tensor and macroyield surface are presented, from which the anisotropic and hardening overall behavior of the composite is proved due to the presence of microresidual stresses.

Additionally, in this paper the approximate two-steps homogenization of composites with wavy inclusions is presented. The method is illustrated through numerical examples of various strain histories and through its comparison with one semianalytical and one computational homogenization method. The comparison results with the Finite Volume Direct Averaging Method are satisfactory in the elastic behavior, as well as for all strain-paths studied in the elastic-plastic behavior, except for the case of shear straining. For this case, the proposed method overestimates the effective hardening behavior, due to the assumed stress uniformity for fixed $x_{2}$. Homogenization approaches based on the dissipation inequality are numerous. However, the present contribution combines homogenization of a wavy medium and homogenization with elastic-perfectly plastic component. The limitations of this method are related to the difficulty of handling arbitrary generalized periodic functions in a three-dimensional setting. 




(a)

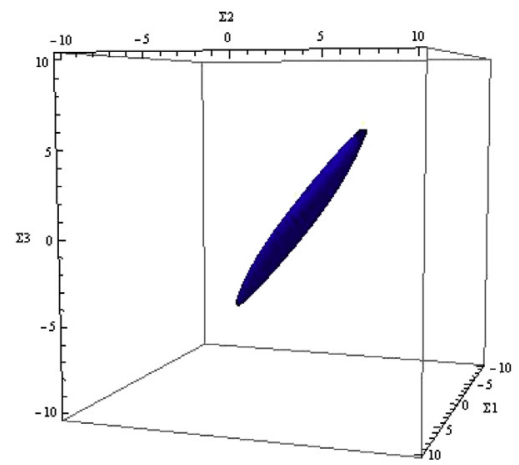

(d)

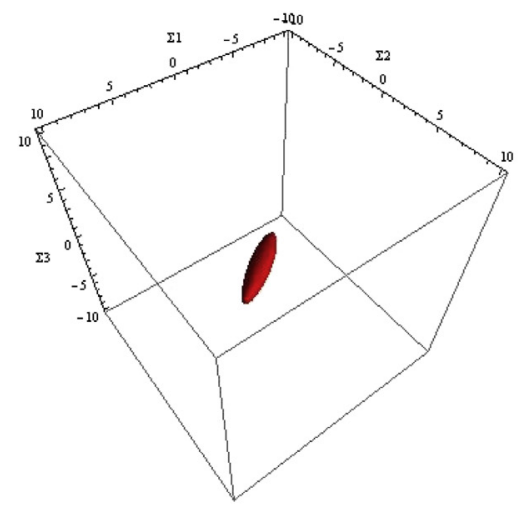

(b)

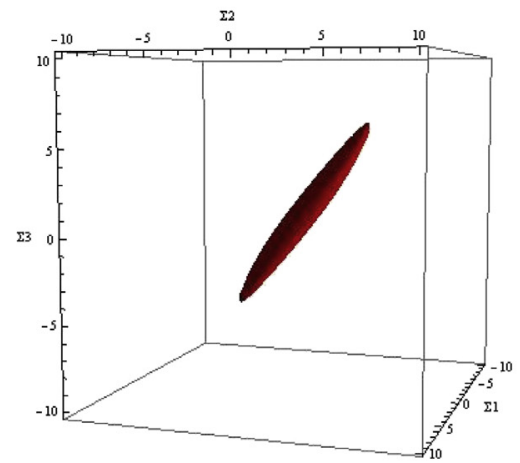

(e)

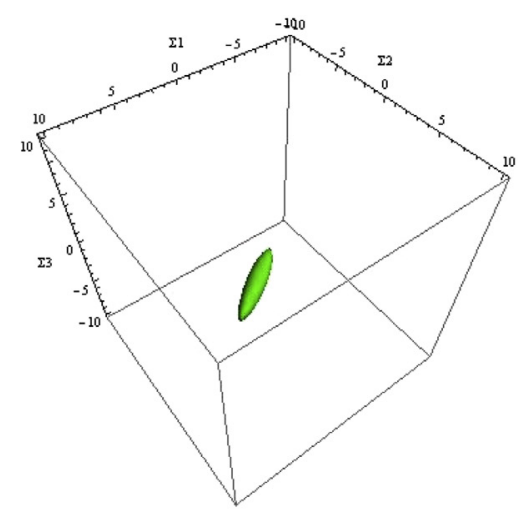

(c)

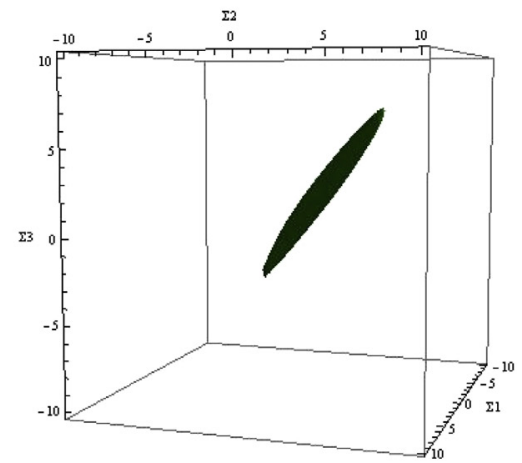

(f)

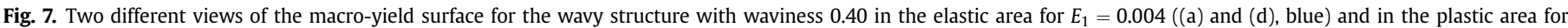

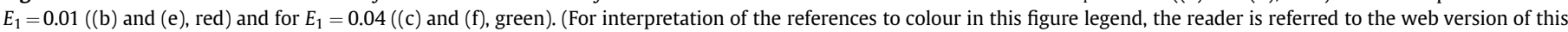
article.)

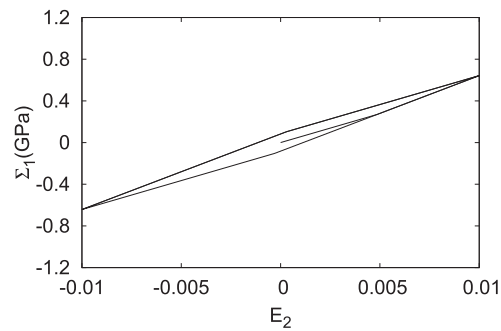

(a)

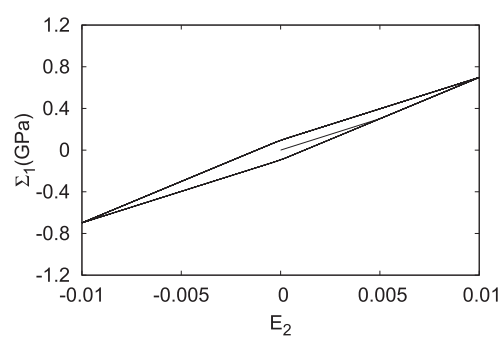

(d)

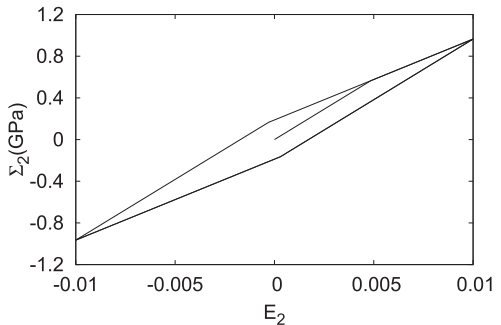

(b)

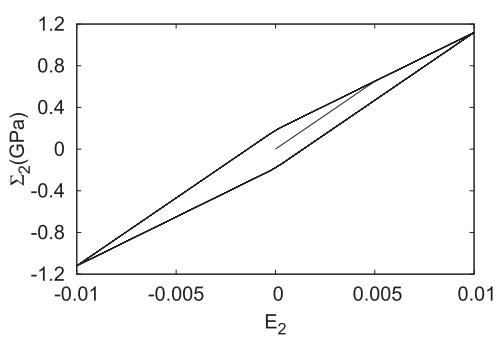

(e)

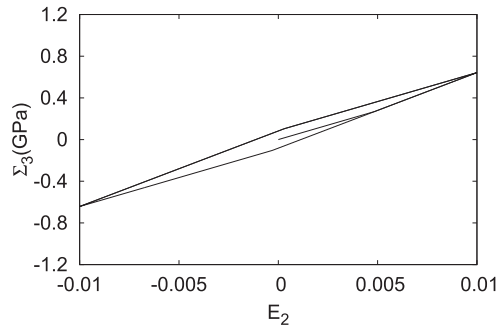

(c)

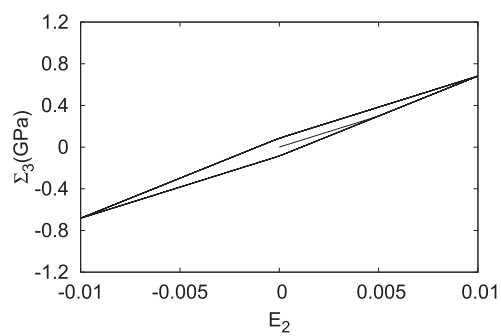

(f)

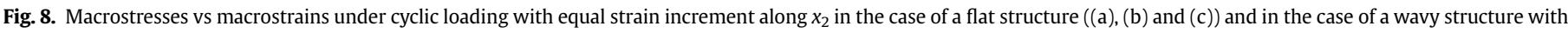
waviness 0.40 ((d), (e) and (f)). 


\section{Acknowledgment}

The authors express their gratitude to Professor Marek-Jerzy Pindera and Mr Wenqiong Tu for many discussions and for providing the FVDAM (Finite Volume Direct Averaging Micromechanics) code used in the numerical experiments of this paper. The theoretical part of this paper is written in the Laboratory of Continuum Mechanics, Technische Universitat, Darmstadt, whose financial support and warm hospitality is gratefully recognized by Nicolas Charalambakis. Many thanks are due to Dr Carsten Broese, Technische Universitat, for many helpful discussions during the stay of the last author in Darmstadt.

\section{Appendix A. The system of Eq. (3.20)}

$$
\begin{aligned}
& \left(G_{E}^{I}\right)_{21}=A_{21} \bar{\alpha}_{11} \tan \theta+A_{22} \bar{\alpha}_{12} \\
& \left(G_{E}^{I}\right)_{22}=1+A_{21} \bar{\alpha}_{12} \tan \theta+A_{22} \bar{\alpha}_{11} \\
& \left(G_{E}^{I}\right)_{23}=A_{21} \bar{\alpha}_{12} \tan \theta+A_{22} \bar{\alpha}_{12} \\
& \left(G_{E}^{I}\right)_{24}=\left(G_{E}^{I}\right)_{25}=0 \\
& \left(G_{E}^{I}\right)_{26}=-\left(A_{21} \bar{\alpha}_{44}+A_{22} \bar{\alpha}_{44} \tan \theta\right)
\end{aligned}
$$

Using the following substitutions,

$\tilde{\alpha}_{i j}=C_{i j}^{I}+\frac{c^{I}}{c^{I I}} C_{i j}^{I I}, \quad \bar{\alpha}_{i j}=C_{i j}^{I}-C_{i j}^{I I}$,

we have for the material I

$\left.M^{I}=\begin{array}{cc}\tilde{\alpha}_{44}+\tilde{\alpha}_{11}(\tan \theta)^{2} & \alpha_{11}(\tan \theta)^{3}-\tilde{\alpha}_{12} \tan \theta \\ \tilde{\alpha}_{12} \tan \theta+\tilde{\alpha}_{44} \tan \theta & \alpha_{12}(\tan \theta)^{2}-\tilde{\alpha}_{11}\end{array}\right)$

$$
\begin{aligned}
& \left(G_{E}^{I}\right)_{31}=\left(G_{E}^{I}\right)_{32}=\left(G_{E}^{I}\right)_{34}=\left(G_{E}^{I}\right)_{35}=\left(G_{E}^{I}\right)_{36}=0 \\
& \left(G_{E}^{I}\right)_{33}=1
\end{aligned}
$$

$$
\begin{aligned}
& \left(G_{E}^{I}\right)_{41}=\left(G_{E}^{I}\right)_{42}=\left(G_{E}^{I}\right)_{43}=\left(G_{E}^{I}\right)_{46}=0 \\
& \left(G_{E}^{I}\right)_{44}=1-\frac{C_{44}^{I}-C_{44}^{I I}}{C_{1}}
\end{aligned}
$$

and

$$
\left(G_{E}^{I}\right)_{45}=\frac{C_{44}^{I}-C_{44}^{I I}}{C_{1}} \tan \theta
$$

\section{Appendix B. Matrices $G_{E}^{I}$ and $G_{E}^{I I}$}

Using the substitutions

$$
\begin{aligned}
& A_{11}(\theta)=\frac{\tilde{\alpha}_{12} \tan ^{2} \theta-\tilde{\alpha}_{11}}{\operatorname{det} M^{I}}, \quad A_{12}(\theta)=\frac{-\tilde{\alpha}_{11} \tan ^{3} \theta+\tilde{\alpha}_{12} \tan \theta}{\operatorname{det} M^{I}}, \\
& A_{21}(\theta)=\frac{-\tilde{\alpha}_{44} \tan \theta-\tilde{\alpha}_{12} \tan \theta}{\operatorname{det} M^{I}}, \quad A_{22}(\theta)=\frac{\tilde{\alpha}_{44}+\tilde{\alpha}_{11} \tan ^{2} \theta}{\operatorname{det} M^{I}}, \\
& C_{1}=\left(1+(\tan \theta)^{2}\right)\left(C_{44}^{I}+\frac{c^{I}}{c^{I I}} C_{44}^{I I}\right),
\end{aligned}
$$

$$
\begin{aligned}
& \left(G_{E}^{I}\right)_{51}=\left(G_{E}^{I}\right)_{52}=\left(G_{E}^{I}\right)_{53}=\left(G_{E}^{I}\right)_{56}=0 \\
& \left(G_{E}^{I}\right)_{54}=\frac{C_{44}^{I}-C_{44}^{I I}}{C_{1}} \tan \theta \\
& \left(G_{E}^{I}\right)_{55}=1-\frac{C_{44}^{I}-C_{44}^{I I}}{C_{1}} \tan ^{2} \theta
\end{aligned}
$$

and those introduced in Appendix A we have

Matrix $G_{E}^{I}$

$$
\begin{aligned}
& \left(G_{E}^{I}\right)_{11}=1-\tan ^{2} \theta\left(A_{21} \bar{\alpha}_{11} \tan \theta+A_{22} \bar{\alpha}_{12}\right)-\tan \theta\left(A_{11} \bar{\alpha}_{11} \tan \theta+A_{12} \bar{\alpha}_{12}\right) \\
& \left(G_{E}^{I}\right)_{12}=-\tan ^{2} \theta\left(A_{21} \bar{\alpha}_{12} \tan \theta+A_{22} \bar{\alpha}_{11}\right)-\tan \theta\left(A_{11} \bar{\alpha}_{12} \tan \theta+A_{12} \bar{\alpha}_{11}\right) \\
& \left(G_{E}^{I}\right)_{13}=-\tan ^{2} \theta\left(A_{21} \bar{\alpha}_{12} \tan \theta+A_{22} \bar{\alpha}_{12}\right)-\tan \theta\left(A_{11} \bar{\alpha}_{12} \tan \theta+A_{12} \bar{\alpha}_{12}\right) \\
& \left(G_{E}^{I}\right)_{14}=\left(G_{E}^{I}\right)_{15}=0 \\
& \left(G_{E}^{I}\right)_{16}=\tan ^{2} \theta\left(A_{21} \bar{\alpha}_{44}+A_{22} \bar{\alpha}_{44} \tan \theta\right)+\tan \theta\left(A_{11} \bar{\alpha}_{44}+A_{12} \bar{\alpha}_{44} \tan \theta\right)
\end{aligned}
$$


$\left(G_{E}^{I}\right)_{61}=A_{11} \bar{\alpha}_{11} \tan \theta+A_{12} \bar{\alpha}_{12}$

$\left(G_{E}^{I}\right)_{62}=A_{11} \bar{\alpha}_{12} \tan \theta+A_{12} \bar{\alpha}_{11}$

$\left(G_{E}^{I}\right)_{63}=A_{11} \bar{\alpha}_{12} \tan \theta+A_{12} \bar{\alpha}_{12}$

$\left(G_{E}^{I}\right)_{64}=\left(G_{E}^{I}\right)_{65}=0$

$\left(G_{E}^{I}\right)_{66}=1-\left(A_{11} \bar{\alpha}_{44}+A_{12} \bar{\alpha}_{44} \tan \theta\right)$

Matrix $G_{E}^{I I}$
$\left(G_{E}^{I I}\right)_{41}=\left(G_{E}^{I I}\right)_{42}=\left(G_{E}^{I I}\right)_{43}=\left(G_{E}^{I I}\right)_{46}=0$

$\left(G_{E}^{I I}\right)_{44}=1-\left(-\frac{c_{I}}{c_{I I}}\right)\left(\frac{C_{44}^{I}-C_{44}^{I I}}{C_{1}}\right)$

$\left(G_{E}^{I I}\right)_{45}=\left(-\frac{c_{I}}{C_{I I}}\right)\left(\frac{C_{44}^{I}-C_{44}^{I I}}{C_{1}} \tan \theta\right)$

$\left(G_{E}^{I I}\right)_{11}=1-\left(-\frac{c_{I}}{c_{I I}}\right)\left(\tan ^{2} \theta\left(A_{21} \bar{\alpha}_{11} \tan \theta+A_{22} \bar{\alpha}_{12}\right)\right)-\left(-\frac{c_{I}}{c_{I I}}\right)\left(\tan \theta\left(A_{11} \bar{\alpha}_{11} \tan \theta+A_{12} \bar{\alpha}_{12}\right)\right)$

$\left(G_{E}^{I I}\right)_{12}=-\left(-\frac{c_{I}}{c_{I I}}\right)\left(\tan ^{2} \theta\left(A_{21} \bar{\alpha}_{12} \tan \theta+A_{22} \bar{\alpha}_{11}\right)\right)-\left(-\frac{c_{I}}{c_{I I}}\right)\left(\tan \theta\left(A_{11} \bar{\alpha}_{12} \tan \theta+A_{12} \bar{\alpha}_{11}\right)\right)$

$\left(G_{E}^{I I}\right)_{13}=-\left(-\frac{c_{I}}{c_{I I}}\right)\left(\tan ^{2} \theta\left(A_{21} \bar{\alpha}_{12} \tan \theta+A_{22} \bar{\alpha}_{12}\right)\right)-\left(-\frac{c_{I}}{c_{I I}}\right)\left(\tan \theta\left(A_{11} \bar{\alpha}_{12} \tan \theta+A_{12} \bar{\alpha}_{12}\right)\right)$

$\left(G_{E}^{I I}\right)_{14}=\left(G_{E}^{I I}\right)_{15}=0$

$\left(G_{E}^{I I}\right)_{16}=\left(-\frac{c_{I}}{c_{I I}}\right)\left(\tan ^{2} \theta\left(A_{21} \bar{\alpha}_{44}+A_{22} \bar{\alpha}_{44} \tan \theta\right)\right)+\left(-\frac{c_{I}}{c_{I I}}\right)\left(\tan \theta\left(A_{11} \bar{\alpha}_{44}+A_{12} \bar{\alpha}_{44} \tan \theta\right)\right)$

$\left(G_{E}^{I I}\right)_{21}=\left(-\frac{c_{I}}{C_{I I}}\right)\left(A_{21} \bar{\alpha}_{11} \tan \theta+A_{22} \bar{\alpha}_{12}\right)$

$\left(G_{E}^{I I}\right)_{22}=1+\left(-\frac{c_{I}}{C_{I I}}\right)\left(A_{21} \bar{\alpha}_{12} \tan \theta+A_{22} \bar{\alpha}_{11}\right)$

$\left(G_{E}^{I I}\right)_{23}=\left(-\frac{c_{I}}{c_{I I}}\right)\left(A_{21} \bar{\alpha}_{12} \tan \theta+A_{22} \bar{\alpha}_{12}\right)$

$\left(G_{E}^{I I}\right)_{24}=\left(G_{E}^{I I}\right)_{25}=0$

$\left(G_{E}^{I I}\right)_{26}=-\left(-\frac{c_{I}}{c_{I I}}\right)\left(A_{21} \bar{\alpha}_{44}+A_{22} \bar{\alpha}_{44} \tan \theta\right)$
$\left(G_{E}^{I I}\right)_{51}=\left(G_{E}^{I I}\right)_{52}=\left(G_{E}^{I I}\right)_{53}=\left(G_{E}^{I I}\right)_{56}=0$

$\left(G_{E}^{I I}\right)_{54}=\left(-\frac{c_{I}}{C_{I I}}\right)\left(\frac{C_{44}^{I}-C_{44}^{I I}}{C_{1}} \tan \theta\right)$

$\left(G_{E}^{I I}\right)_{55}=1-\left(-\frac{C_{I}}{C_{I I}}\right)\left(\frac{C_{44}^{I}-C_{44}^{I I}}{C_{1}} \tan ^{2} \theta\right)$

$\left(G_{E}^{I I}\right)_{61}=\left(-\frac{c_{I}}{c_{I I}}\right)\left(A_{11} \bar{\alpha}_{11} \tan \theta+A_{12} \bar{\alpha}_{12}\right)$

$\left(G_{E}^{I I}\right)_{62}=\left(-\frac{c_{I}}{c_{I I}}\right)\left(A_{11} \bar{\alpha}_{12} \tan \theta+A_{12} \bar{\alpha}_{11}\right)$

$\left(G_{E}^{I I}\right)_{63}=\left(-\frac{c_{I}}{c_{I I}}\right)\left(A_{11} \bar{\alpha}_{12} \tan \theta+A_{12} \bar{\alpha}_{12}\right)$

$\left(G_{E}^{I I}\right)_{64}=\left(G_{E}^{I I}\right)_{65}=0$

$\left(G_{E}^{I I}\right)_{66}=1-\left(-\frac{c_{I}}{c_{I I}}\right)\left(A_{11} \bar{\alpha}_{44}+A_{12} \bar{\alpha}_{44} \tan \theta\right)$

Appendix C. Matrices $G_{I}^{I}, G_{I}^{I I}, G_{I I}^{I}, G_{I I}^{I I}$

Matrix $G_{I}^{I}$

$\left(G_{I}^{I}\right)_{11}=\tan \theta\left(A_{11} C_{11}^{I} \tan \theta+A_{12} C_{12}^{I}\right)+\tan ^{2} \theta\left(A_{21} C_{11}^{I} \tan \theta+A_{22} C_{12}^{I}\right)$

$\left(G_{I}^{I}\right)_{12}=\tan \theta\left(A_{11} C_{12}^{I} \tan \theta+A_{12} C_{11}^{I}\right)+\tan ^{2} \theta\left(A_{12} C_{12}^{I} \tan \theta+A_{22} C_{11}^{I}\right)$

$\left(G_{I}^{I}\right)_{13}=\tan \theta\left(A_{11} C_{12}^{I} \tan \theta+A_{12} C_{12}^{I}\right)+\tan ^{2} \theta\left(A_{21} C_{12}^{I} \tan \theta+A_{22} C_{12}^{I}\right)$

$\left(G_{I}^{I}\right)_{14}=\left(G_{I}^{I}\right)_{15}=0$

$\left(G_{I}^{I}\right)_{16}=-\tan \theta\left(A_{11} C_{44}^{I}+A_{12} C_{44}^{I} \tan \theta\right)-\tan ^{2} \theta\left(A_{21} C_{44}^{I}+A_{22} C_{44}^{I} \tan \theta\right)$ 
$\left(G_{I}^{I}\right)_{21}=-\left(A_{21} C_{11}^{I} \tan \theta+A_{22} C_{12}^{I}\right)$

$\left(G_{I}^{I}\right)_{22}=-\left(A_{21} C_{12}^{I} \tan \theta+A_{22} C_{11}^{I}\right)$

$\left(G_{I}^{I}\right)_{23}=-\left(A_{21} C_{12}^{I} \tan \theta+A_{22} C_{12}^{I}\right)$

(C.2)

$\left(G_{I}^{I}\right)_{24}=\left(G_{I}^{I}\right)_{25}=0$

$\left(G_{I}^{I}\right)_{26}=A_{21} C_{44}^{I}+A_{22} C_{44}^{I} \tan \theta$

$\left(G_{I}^{I}\right)_{31}=\left(G_{I}^{I}\right)_{32}=\left(G_{I}^{I}\right)_{33}=\left(G_{I}^{I}\right)_{34}=\left(G_{I}^{I}\right)_{35}=\left(G_{I}^{I}\right)_{36}=0$

$\left(G_{I}^{I}\right)_{41}=\left(G_{I}^{I}\right)_{42}=\left(G_{I}^{I}\right)_{43}=\left(G_{I}^{I}\right)_{46}=0$

$\left(G_{I}^{I}\right)_{44}=\frac{C_{44}^{I}}{C_{1}}$

$\left(G_{I}^{I}\right)_{45}=-\frac{C_{44}^{I}}{C_{1}} \tan \theta$

$\left(G_{I}^{I}\right)_{51}=\left(G_{I}^{I}\right)_{52}=\left(G_{I}^{I}\right)_{53}=\left(G_{I}^{I}\right)_{56}=0$

$\left(G_{I}^{I}\right)_{54}=-\frac{C_{44}^{I}}{C_{1}} \tan \theta$

$\left(G_{I}^{I}\right)_{55}=\frac{C_{44}^{I}}{C_{1}} \tan ^{2} \theta$

$\left(G_{I}^{I}\right)_{61}=-\left(A_{11} C_{11}^{I} \tan \theta+A_{12} C_{12}^{I}\right)$

$\left(G_{I}^{I}\right)_{62}=-\left(A_{11} C_{12}^{I} \tan \theta+A_{12} C_{11}^{I}\right)$

$\left(G_{I}^{I}\right)_{63}=-\left(A_{11} C_{12}^{I} \tan \theta+A_{12} C_{12}^{I}\right)$

$\left(G_{I}^{I}\right)_{64}=\left(G_{I}^{I}\right)_{65}=0$

$\left(G_{I}^{I}\right)_{66}=A_{11} C_{44}^{I}+A_{12} C_{44}^{I} \tan \theta$

Matrix $G_{I I}^{I}$

$$
\left(G_{I I}^{I}\right)_{31}=\left(G_{I I}^{I}\right)_{32}=\left(G_{I I}^{I}\right)_{33}=\left(G_{I I}^{I}\right)_{34}=\left(G_{I I}^{I}\right)_{35}=\left(G_{I I}^{I}\right)_{36}=0
$$

$\left(G_{I I}^{I}\right)_{41}=\left(G_{I I}^{I}\right)_{42}=\left(G_{I I}^{I}\right)_{43}=\left(G_{I I}^{I}\right)_{46}=0$

$\left(G_{I I}^{I}\right)_{44}=-\frac{C_{44}^{I I}}{C_{1}}$

$\left(G_{I I}^{I}\right)_{45}=\frac{C_{44}^{I I}}{C_{1}} \tan \theta$

$\left(G_{I I}^{I}\right)_{51}=\left(G_{I I}^{I}\right)_{52}=\left(G_{I I}^{I}\right)_{53}=\left(G_{I I}^{I}\right)_{56}=0$

$\left(G_{I I}^{I}\right)_{54}=\frac{C_{44}^{I I}}{C_{1}} \tan \theta$

$\left(G_{I I}^{I}\right)_{55}=-\frac{C_{44}^{I I}}{C_{1}} \tan ^{2} \theta$

$\left(G_{I I}^{I}\right)_{61}=A_{11} C_{11}^{I I} \tan \theta+A_{12} C_{12}^{I I}$

$\left(G_{I I}^{I}\right)_{62}=A_{11} C_{12}^{I I} \tan \theta+A_{12} C_{11}^{I I}$

$\left(G_{I I}^{I}\right)_{63}=A_{11} C_{12}^{I I} \tan \theta+A_{12} C_{12}^{I I}$

$\left(G_{I I}^{I}\right)_{64}=\left(G_{I I}^{I}\right)_{65}=0$

$\left(G_{I I}^{I}\right)_{66}=-\left(A_{11} C_{44}^{I I}+A_{12} C_{44}^{I I} \tan \theta\right)$

Matrices $G_{I}^{I I}$ and $G_{I I}^{I I}$

$G_{I}^{I I}=\left(-\frac{c^{I}}{c^{I I}}\right) G_{I}^{I}$

and

$G_{I I}^{I I}=\left(-\frac{c^{I}}{c^{I I}}\right) G_{I I}^{I}$

$\left(G_{I I}^{I}\right)_{11}=-\tan \theta\left(A_{11} C_{11}^{I I} \tan \theta+A_{12} C_{12}^{I I}\right)-\tan ^{2} \theta\left(A_{21} C_{11}^{I I} \tan \theta+A_{22} C_{12}^{I I}\right)$

$\left(G_{I I}^{I}\right)_{12}=-\tan \theta\left(A_{11} C_{12}^{I I} \tan \theta+A_{12} C_{11}^{I I}\right)-\tan ^{2} \theta\left(A_{21} C_{12}^{I I} \tan \theta+A_{22} C_{11}^{I I}\right)$

$\left(G_{I I}^{I}\right)_{13}=-\tan \theta\left(A_{11} C_{12}^{I I} \tan \theta+A_{12} C_{12}^{I I}\right)-\tan ^{2} \theta\left(A_{21} C_{12}^{I I} \tan \theta+A_{22} C_{12}^{I I}\right)$

$\left(G_{I I}^{I}\right)_{14}=\left(G_{I I}^{I}\right)_{15}=0$

$\left(G_{I I}^{I}\right)_{16}=\tan \theta\left(A_{11} C_{44}^{I I}+A_{12} C_{44}^{I I} \tan \theta\right)+\tan ^{2} \theta\left(A_{21} C_{44}^{I I}+A_{22} C_{44}^{I I} \tan \theta\right)$

\section{References}

$\left(G_{I I}^{I}\right)_{21}=A_{21} C_{11}^{I I} \tan \theta+A_{22} C_{12}^{I I}$

$\left(G_{I I}^{I}\right)_{22}=A_{21} C_{12}^{I I} \tan \theta+A_{22} C_{11}^{I I}$

$\left(G_{I I}^{I}\right)_{23}=A_{21} C_{12}^{I I} \tan \theta+A_{22} C_{12}^{I I}$

$\left(G_{I I}^{I}\right)_{24}=\left(G_{I I}^{I}\right)_{25}=0$

$\left(G_{I I}^{I}\right)_{26}=-\left(A_{21} C_{44}^{I I}+A_{22} C_{44}^{I I} \tan \theta\right)$
[1] Alber H-D. Materials with memory. Lecture notes in mathematics, vol. 1682. Berlin: Springer-Verlag; 1998.

[2] Alber H-D. Evolving microstructure and homogenization. Continuum Mech Thermodyn 2000;12(4):235-86.

[3] Allaire G. Homogenization and two-scale convergence. SIAM J Math Analysis 1992;23:1482-518.

[4] Balokhonov R, Romanova V, Schmauder S, Martynov S, Kovalevskaya Z. A mesomechanical analysis of plastic strain and fracture localization in a material with a bilayer coating. Compos Part B 2014;66:276-86.

[5] Bensoussan A, Lions J-L, Papanicolaou G. Asymptotic methods for periodic structures. North Holland. 1978. 
[6] Birman V, Chandrashekhara K, Hopkins M, Volz J. Strength analysis of particulate polymers. Compos Part B 2013;54:278-88.

[7] Briane M. Homogénéisation de matériaux fibrés [Ph.D. thesis]. Paris VI: Université Pierre et Marie Curie; 1990.

[8] Briane M. Three models of non periodic fibrous materials obtained by homogenization. RAIRO-Modélisation mathématique et analyse numérique $1993 ; 27: 759-75$

[9] Cavalcante M, Marques S, Pindera M. Transient thermomechanical analysis of a layered cylinder by the parametric finite-volume theory. J Therm Stresses 2009;32:112-34.

[10] Chaboche J, Kanoute P, Ross A. On the capabilities of mean field approaches for the description of plasticity in metal matrix composites. Int J Plasticity 2005;21:1409-34.

[11] Chatzigeorgiou G, Charalambakis N. Instability analysis of non-homogeneous materials under biaxial loading. Int J Plasticity 2005;21:1970-99.

[12] Chatzigeorgiou G, Charalambakis N, Murat F. Homogenization problems of a hollow cylinder made of elastic materials with discontinuous properties. Int J Solids Struct 2008;45:5165-80.

[13] Chatzigeorgiou G, Charalambakis N, Murat F. Homogenization of a pressurized tube made of elastoplastic materials with discontinuous properties. Int ] Solids Struct 2009;46:3902-13.

[14] Chatzigeorgiou G, Efendiev Y, Charalambakis N, Lagoudas D. Effective thermoelastic properties of composites with periodicity in cylindrical coordinates. Int J Solids Struct 2012;49:2590-603.

[15] Chatzigeorgiou G, Efendiev Y, Lagoudas DC. Homogenization of aligned "fuzzy fiber" composites. Int J Solids Struct 2011;48(19):2668-80.

[16] Chatzigeorgiou G, Kalpakides V, Charalambakis N. Biaxial loading of continuously graded thermoviscoplastic materials. Comput Mech 2007;39:335-55.

[17] Dvorak G. Transformation field analysis of inelastic composite materials. Proc R Soc Lond A 1992:437:311-27.

[18] Dvorak G, Bahel-El-Din Y, Wafa A. Implementation of the transformation field analysis for inelastic composite materials. Comput Mech 1994;14:201-28.

[19] Fish J, Shek K, Pandheeradi M, Shephard M. Computational plasticity for composite structures based on mathematical homogenization. theory and practice. Comput Methods Appl Mech Eng 1997;148:53-73.

[20] Francfort G, Suquet P. Homogenization and mechanical dissipation in thermoviscoelasticity. Archive Ration Mech Analysis 1986;96:268-93.

[21] Germain P. Sur certaines definitions liees a l'energie en mecanique des solides, Int J Eng Sci 1982;20:245-59.

[22] Katz A, Trinh C, Wright J, Tu W, Pindera M. Plastic strain localization in periodic materials with wavy brick-and-mortar architectures and its effect on the homogenized response. Compos Part B 2015;68:270-80.

[23] Khatam H, Pindera M. Parametric finite-volume micromechanics of periodic materials with elastoplastic phases. Int J Plasticity 2009a;25:1386-411.

[24] Khatam H, Pindera M. Thermoelastic moduli of periodic multilayers with wavy microstructures. Compos Part B 2009b;40:50-64.

[25] Khatam H, Pindera M. Plasticity-triggered architectural effects in periodic multilayers with wavy microstructures. Int J Plasticity 2010;26:273-87.

[26] Khatam H, Pindera M. Microstructural scale effects in the nonlinear elastic response of bio-inspired wavy multilayers undergoing finite deformation. Compos Part B 2012;43:869-84.

[27] Kundalwal S, Ray M. Effect of carbon nanotubes waviness on the effective thermoelastic properties of a novel continuous fuzzy fiber-reinforced composite. Compos Part B 2014a;57:199-209.

[28] Kundalwal S, Ray M. Improved thermoelastic coefficients of a novel short fuzzy fiber-reinforced composite with wavy carbon nanotubes. J Mech Mater Struct 2014b;9:1-25.

[29] Love B, Batra R. Determination of effective thermomechanical parameters of a mixture of two elastothermoviscoplastic constituents. Int J Plasticity 2006;22: 1026-61.

[30] Michel J, Galvanetto U, Suquet P. Constitutive relations involving internal variables based on a micromechanical analysis. In: Maugin G, Drouot $\mathrm{R}$, Sidoroff F, editors. Continuum thermomechanics: the art and science of modelling material behaviour. Kluwer Acad. Pub; 2000.

[31] Michel J, Moulinec H, Suquet P. Effective properties of composite materials with periodic microstructure: a computational approach. Comput Methods Appl Mech Eng 1999;172:109-43.
[32] Michel J, Suquet P. Computational analysis of nonlinear composites structures using the nonuniform transformation field analysis. Comput Methods Appl Mech Eng 2004;193:5477-502.

[33] Michel J, Suquet P. Nonuniform transformation field analysis: a reduced model for multiscale nonlinear problemes in solid mechanics. In: Galvanetto U, Aliabadi F, editors. Multiscale modelling in solid mechanicscomputational approaches; 2009.

[34] Moulinec H, Suquet P. Intraphase strain heterogeneity in nonlinear composites: a computational approach. Eur J Mech A Solids 2003;22:751-70.

[35] Murat F, Tartar L. H-convergence. In: Cherkaev A, Kohn RV, editors. Topics in the mathematical modelling of composite materials. Progress in nonlinear differential equations and their applications, vol. 31. Birkhäuser; 1997. p. $21-43$.

[36] Nackenhorst U, Kardas D, Helmich T, Lenz C, Shan W. Computational techniques for multiscale analysis of materials and interfaces. Lect Notes Appl Comput Mech 2011;57:133-67.

[37] Nguyen QS. Mechanical modelling of anelasticity. Rev Phys Appl 1988;23: 325-30.

[38] Nie G, Batra R. Exact solutions and material tailoring for functionally graded hollow circular cylinders. J Elast 2010:99:179-201.

[39] Nie G, Zhong Z, Batra R. Material tailoring for functionally graded hollow cylinders and spheres. Compos Sci Technol 2011;71:666-73.

40] Pindera M, Charalambakis N, Lagoudas D, Hui D. Special issue of composites part b: homogenization and micromechanics of smart and multifunctional materials. Compos Part B 2012;43:2493-4.

41] Pindera M, Khatam H, Drago S, Bansal Y. Micromechanics of spatially uniform heterogeneous media: a critical review and emerging approaches. Compos Part B 2009; 40:349-78.

[42] Ponte-Castaneda P, Suquet P. Nonlinear composites. Adv Appl Mech 1997;34 $171-302$.

[43] Pruchnicki E, Shahrour I. A macroscopic elastoplastic constitutive law for multilayered media: application to reinforced earth material. Int J Solids Struct 1994; 18:507-18.

[44] Qu J, Cherkaoui M. Fundamentals of micromechanics of solids. John Wiley \& Sons; 2006.

[45] Seidel G, Chatzigeorgiou G, Ren X, Lagoudas D. Multiscale modeling of multifunctonal fuzzy fibers based on multi-walled carbon nanotubes. Springer Ser Mater Sci 2014:188:135-76.

[46] Simo JC, Hughes TJR. Computational inelasticity. Springer-Verlag; 1998.

[47] Suquet P. Overall potentials and extremal surfaces of power law or ideally plastic composites. J Mech Phys Solids 1993;41:981-1002.

[48] Suquet PM. Elements of homogenization for inelastic solid mechanics [Tech. Repp. 85-2]. Montpellier: Université des Sciences et Techniques du Languedoc; July 1985.

[49] Suquet PM. Elements of homogenization for inelastic solid mechanics. In: Sanchez-Palencia E, Zaoui A, editors. Homogenization techniques for composite materials, Lecture notes in physics 272. Springer Verlag; 1987. p. $193-278$.

[50] Tartar L. Nonlinear constitutive relations and homogenization. In: Contemporary developments in continuum mechanics and partial differential equations. North-Holand; 1978.

[51] Terada K, Kikuchi N. A class of general algorithms for multi-scale analyses of heterogeneous media. Comput methods Appl Mech Eng 2001;190: 5427-64.

52] Tsalis D, Baxevanis T, Chatzigeorgiou G, Charalambakis N. Homogenization of elastoplastic composites with generalized periodicity in the microstructure. Int J Plasticity 2013;51:161-87.

[53] Tsalis D, Chatzigeorgiou G, Charalambakis N. Homogenization of structures with generalized periodicity. Compos Part B 2012;43:2495-512.

[54] Tu W, Pindera M. Targeting the finite-deformation response of wavy biolog ical tissues with bio-inspired material architectures. J Mech Behav Biomed Mater 2013:28:291-308.

[55] Vigliotti A, Deshpande V, Pasini D. Non linear constitutive models for lattice materials. J Mech Phys Solids 2014;64:44-60.

[56] Wu Y, Nie Y. Prediction of effective properties for random heterogeneous materials with extrapolation. Archive Appl Mech 2014;84:247-61. 